\title{
Experimental evolution makes microbes more cooperative with their local host genotype
}

\author{
Batstone, R. T., O’Brien, A. M., Harrison, T. L., \& Frederickson, \\ M. E.
}

Version Accepted Manuscript

Citation Batstone, R. T., O’Brien, A. M., Harrison, T. L., \& Frederickson, M. E. (published version) (2020). Experimental evolution makes microbes more cooperative with their local host genotype. Science, 370(6515), 476-478.

DOI http://dx.doi.org/10.1126/science.abb7222

Publisher's Statement This is the author's version of the work. It is posted here by permission of the AAAS for personal use, not for redistribution. The definitive version was published in Science on 10/23/2020, DOI: http://dx.doi.org/10.1126/science.abb7222

How to cite TSpace items

Always cite the published version, so the author(s) will receive recognition through services that track citation counts, e.g. Scopus. If you need to cite the page number of the author manuscript from TSpace because you cannot access the published version, then cite the TSpace version in addition to the published version using the permanent URI (handle) found on the record page.

This article was made openly accessible by $U$ of $T$ Faculty. Please tell us how this access benefits you. Your story matters. 


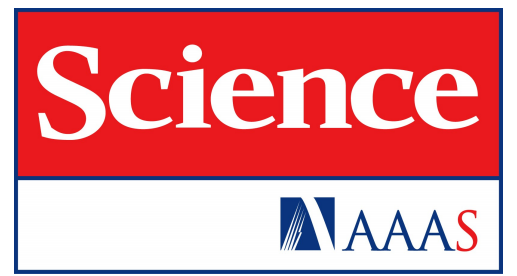

\title{
Experimental evolution makes microbes more cooperative with their local host genotype
}

\author{
Rebecca T. Batstone, ${ }^{1,2, *}$ Anna M. O’Brien, ${ }^{1,3}$ \\ Tia L. Harrison, ${ }^{1} \&$ Megan E. Frederickson ${ }^{1,4,5}$
}

${ }^{1}$ Department of Ecology \& Evolutionary Biology, University of Toronto, Toronto, ON, M5S 3B2, Canada

${ }^{2}$ Carl R. Woese Institute for Genomic Biology, University of Illinois, Urbana, IL, 61801, USA

${ }^{3}$ Department of Mechanical \& Industrial Engineering, University of Toronto, ON, M5S 3G8, Canada

${ }^{4}$ Centre for the Analysis of Genome Evolution \& Function, University of Toronto, ON, M5S 3B2, Canada

${ }^{5}$ Radcliffe Institute for Advanced Study, Harvard University, Cambridge, MA, 02138, USA

*To whom correspondence should be addressed; E-mail: rbats98@illinois.edu.

Advances in microbiome science require a better understanding of how beneficial microbes adapt to hosts. We tested if hosts select for more cooperative microbial strains with a year-long evolution experiment and a cross-inoculation experiment designed to explore how nitrogen-fixing bacteria (rhizobia) adapt to legumes. We paired Ensifer meliloti with one of five Medicago truncatula genotypes that vary in how strongly they 'choose' bacterial symbionts. Independent of host choice, Ensifer meliloti rapidly adapted to its local host genotype and derived microbes were more beneficial when they shared evolutionary history with their host. This local adaptation was mostly limited to the symbiosis plasmids, with mutations in putative signalling genes. Thus, cooperation depends on the match between partner genotypes and increases as bacteria adapt to their local host. 
Host-associated microbiota are often beneficial, but we have a limited understanding of adaptation between partners in these mutualisms, especially at the genomic level. Mutualisms are sometimes viewed as reciprocal parasitism, potentially resulting in antagonistic coevolution that maintains genetic variation within populations. However, recent work has questioned the prevalence of fitness conflict in mutualisms (1), and concordant fitness interests between partners should lead to evolutionary stasis, reducing genetic variation in mutualistic traits like partner quality $(2$, $3)$.

Poor-quality microbes could be 'cheaters' that increase their own fitness at their host's expense (4). However, hosts often 'choose' their microbiota (5) and preferentially associate with or reward more cooperative microbes, selecting against would-be cheaters $(6,7)$. Such partner choice can be adaptive $(8,9)$, but partner choice is also paradoxical: if 'choosy' hosts select high-quality symbionts, variation in symbiont quality should decrease, reducing the selective advantage of partner choice (10). Still, many hosts are 'choosy' and many symbionts are not very beneficial $(4,11)$, making the persistence of ineffective microbes perplexing.

Ineffective microbes might simply be mis-matched with their host. A high-quality microbe on one host genotype may be a low-quality microbe on another. Such genotype-by-genotype (G x G) interactions for symbiont quality are common, maintain variation in the benefits symbionts provide to hosts $(3,12)$, and are a prerequisite for coevolution $(13,14)$. Local adaptation, whereby partners from the same site outperform partners from different sites, can generate $G \times 6$ interactions and occurs in mutualisms $(12,15)$, but is not ubiquitous (16). Furthermore, the genetic mechanisms underlying local adaptation or $\mathrm{G}$ x $\mathrm{G}$ interactions remain largely unknown.

Legume-rhizobium interactions are economically and ecologically important, and a model for studying mutualisms. Legumes trade carbon for nitrogen fixed by rhizobia, which they house in root nodules $(6,9)$. Rhizobia have rapid generation times, can be cultured, and can acquire mutations through horizontal gene transfer (17). Rhizobia are also amenable to genome-wide association studies (GWAS) that can identify genomic variants associated with specific phenotypes (18). Adapting these approaches, Burghardt et al. (19) found stronger selection on rhizobia in hosts 
than when free-living in soil.

Here, we resequenced and cross-inoculated rhizobia after they evolved on one of five host genotypes that vary in 'choosiness' to test whether choosier hosts select for more cooperative symbionts, or whether rhizobia adapt to their local host genotype. We used two rhizobia strains that differ in host benefits: ineffective nitrogen-fixer Ensifer meliloti strain Sm1021 ("Em1021"), and effective nitrogen-fixer E. meliloti strain WSM1022 ("Em1022") (9, 20). We paired both strains with one of five inbred lines of Medicago truncatula: line 270 is indiscriminate, while the others all prefer Em1022 to Em1021, with line 267 almost exclusively partnering with Em1022 in past experiments $(9,20)$. After a year-long evolution experiment spanning five plant generations, we isolated 'derived' rhizobia from nodules and compared them to the original 'ancestral' strains.

Even though Em1021 started with a two-fold advantage (20), it went nearly extinct on all host lines (Fig. 1). At the end of the experiment, Em1021 associated with only $15 \%$ of plants and occupied only $3 \%$ of 336 sampled nodules. In a linear model that combined strain frequencies in soil and nodules (20), we found a marginally significant main effect of generation $\left(\mathrm{F}_{3,96}=2.40\right.$, $\mathrm{p}$ $=0.0730)$, but no effect of plant line $\left(\mathrm{F}_{4,96}=0.552, \mathrm{p}=0.698\right)$. The effective symbiont, Em1022, outcompeted Em1021 on all hosts regardless of 'choosiness', meaning partner choice was not an important selective force.

We assayed Em1022 phenotypes by planting new seeds of the same five plant lines and singly inoculating each with either ancestral Em1022 or one of 40 derived isolates, testing replicates of all possible combinations of host genotypes and isolates (20). We quantified the symbiotic quality of rhizobium isolates by measuring the aboveground plant biomass, and rhizobium fitness by measuring nodule number. We also sequenced whole genomes of the 40 derived isolates and nine replicates each of the ancestors, and conducted GWAS for symbiont quality and rhizobium fitness both on specific host genotypes and across all plant lines (20).

Variation in the benefits that experimentally evolved bacteria conferred to hosts was largely determined by the match between rhizobium and host genotypes. Derived and ancestral Em1022 did not differ significantly in symbiont quality or fitness (Tables S1A \& S2A). However, derived 


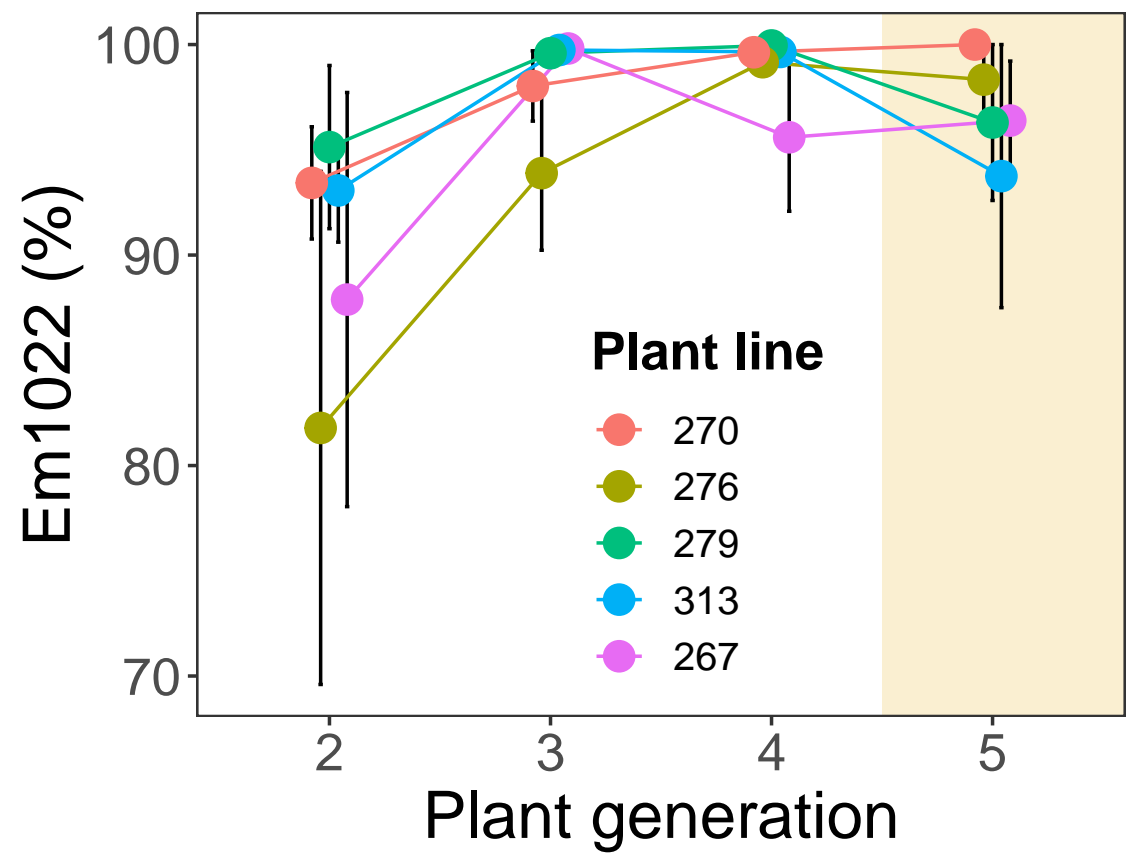

Figure 1: The high-quality microbial partner spread nearly to fixation in all treatments. Percentage (mean $\pm 1 \mathrm{SE}$ ) of the effective $\mathrm{N}$-fixing strain (E. meliloti 1022) across plant generations in the evolution experiment, calculated from soil samples for generations 2-4, and nodules dissected from plants in generation 5 (shaded in yellow). Colors indicate five $M$. truncatula genotypes, as in legend. All plants were initially inoculated with a 2:1 ratio of Em1021:Em1022, or 33\% Em1022.

bacteria provided greater benefits to the host genotype they were paired with during the evolution experiment (Fig. 2A), although a few isolates were high- or low-quality symbionts across plant lines (Figs. S1 \& S2). Derived rhizobia also achieved higher fitness when tested on their local host genotype, with the exception of isolates that evolved on the least 'choosy' host genotype (270, Fig. 2B). Linear mixed models found significantly positive effects of shared evolutionary history on both rhizobium quality and fitness (Tables S1B \& S2B).

Averaged across all hosts, there was a positive relationship between rhizobia quality and fitness (Fig. S3), suggesting that none of the 40 derived strains evolved to 'cheat' and that strong fitness feedbacks make cooperation adaptive for rhizobia. Pairwise correlations between the same trait on different hosts were much weaker and often not statistically significant (Fig. S4), suggesting that microbial quality or fitness on one host is not highly predictive of quality or fitness on another.

Genomic sequencing of E. meliloti isolates uncovered 1330 genetic variants, including 547 

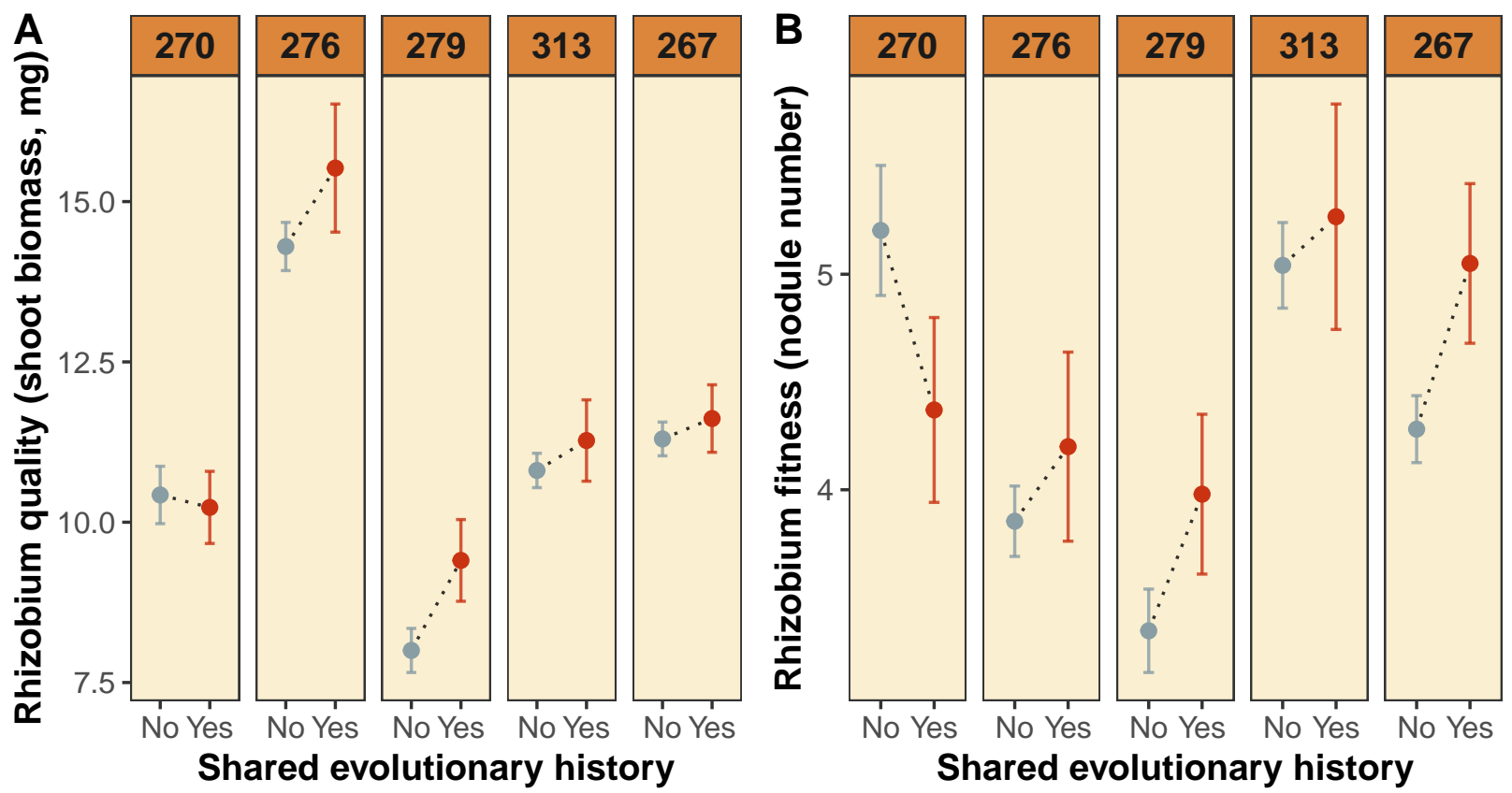

Figure 2: Bacteria adapt to their local host genotype. Mean $\pm 1 \mathrm{SE}$ shoot biomass (A) and nodule number (B) for all possible combinations of 40 derived bacterial isolates from the evolution experiment and 5 plant genotypes (numbered across the top). Bacteria share an evolutionary history with the host genotype they evolved on during the evolution experiment.

de novo mutations (Fig. S5A). Most de novo variants (305) were unique to rhizobia derived on the least choosy host line (270); fewer variants (16-59) were unique to rhizobia derived on other lines (Fig. S5A). After filtering out most singletons and variants in high linkage disequilibrium, reducing our set to 363 variants (Fig. S5B), GWAS identified 145 variants significantly associated with rhizobia quality or fitness in one or more host environments (Table S3). These variants were mostly located on two symbiosis plasmids, pSymA and pSymB, that contain genes essential for the symbiotic and free-living phases of the rhizobium life cycle, respectively $(21,22)$; sixty-two significant variants were on pSymA, 68 on pSymB, and only 15 were on the bacterial chromosome.

Beta scores, which represent the strength of the association between a genomic variant and a phenotype, were strongly positively correlated between symbiont quality and fitness (Fig. 3A), meaning that genomic variants that increased microbial fitness also increased microbial benefits to plants. Within each host environment, most significant variants evolved in isolates paired with that host genotype in the evolution experiment (i.e., local variants; red symbols in Fig. 3A and numbers 
before commas in Fig. 3B). We categorized variant effects as 'cooperative' if beta scores for symbiont quality and fitness were both greater than zero, 'defective' if beta scores were both less than zero, 'altruistic' if beta scores were positive for symbiont quality but negative for symbiont fitness, or 'cheater' if beta scores were positive for symbiont fitness but negative for symbiont quality. In all five host environments, most significant variant effects were cooperative and local (Fig. 3B, Table S3).

We used permutations to assess whether this pattern could have occurred by chance, randomly assigning genomes to phenotypes 1000 times and re-calculating beta scores (20). There were significantly more local-cooperative associations in all five host environments (+ symbols in Fig. 3B) and significantly fewer local-altruistic and local-cheater associations in some host environments (symbols in Fig. 3B) at a 5\% false discovery rate. The numbers of associations in other categories (e.g., defective variants) did not differ from null expectations.

Local, cooperative variants were located in candidate genes putatively encoding a calciumbinding protein, a FAD-binding oxidoreductase, and two Ti-type conjugative transfer relaxases, among others (Table S3). These functions may relate to nodule initiation or rhizobium survival inside nodules (23). Variant effects were often host-specific, generally conditionally beneficial on their local host or deleterious on a non-local host (Table S3), suggesting selection in local and drift in non-local contexts. When variants exhibited significant effects on multiple hosts, they tended to be consistently beneficial or deleterious across hosts; only two variants had significant, opposite-sign effects across hosts, suggesting trade-offs are rare (Table S3). In summary, cooperation evolved more often, and cheating or altruism less often, than expected by chance, as bacteria adapted to their local host genotype.

In our evolution experiment, we expected the 'choosiest' host line to most strongly favor the better symbiont, Em1022. However, the ineffective strain, Em1021, went nearly extinct across all plant lines, even on indiscriminate hosts (e.g., 270, Fig. 1), suggesting this strain is a universally poor competitor. In contrast, Em1022 evolved novel variants that differed in both symbiont quality and fitness. Derived bacteria provided greater host benefits and generally achieved higher fitness 
A
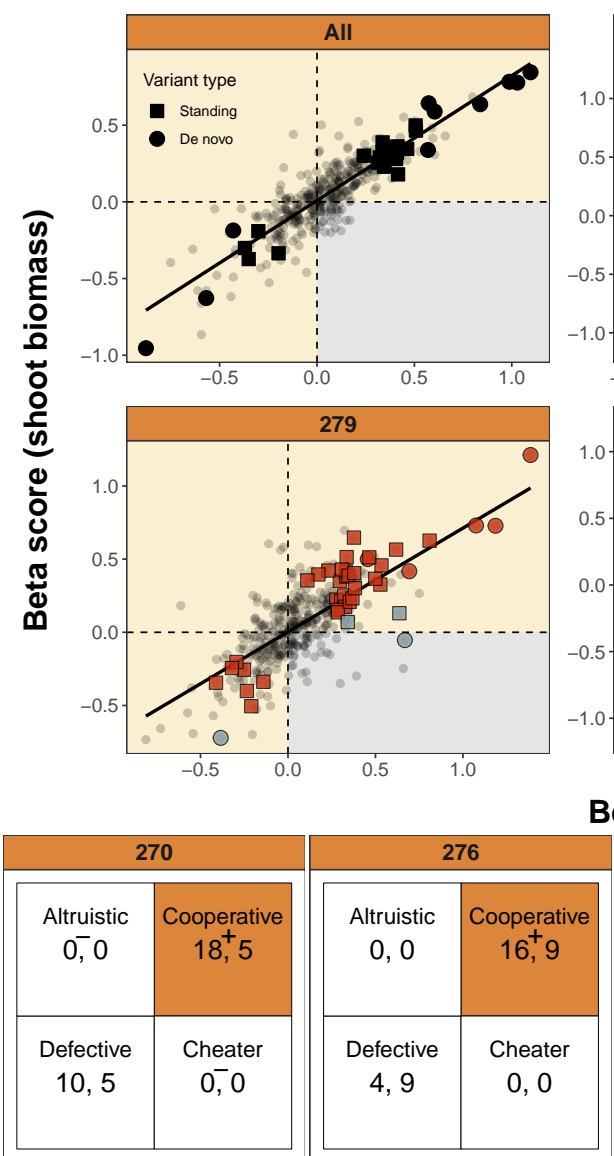
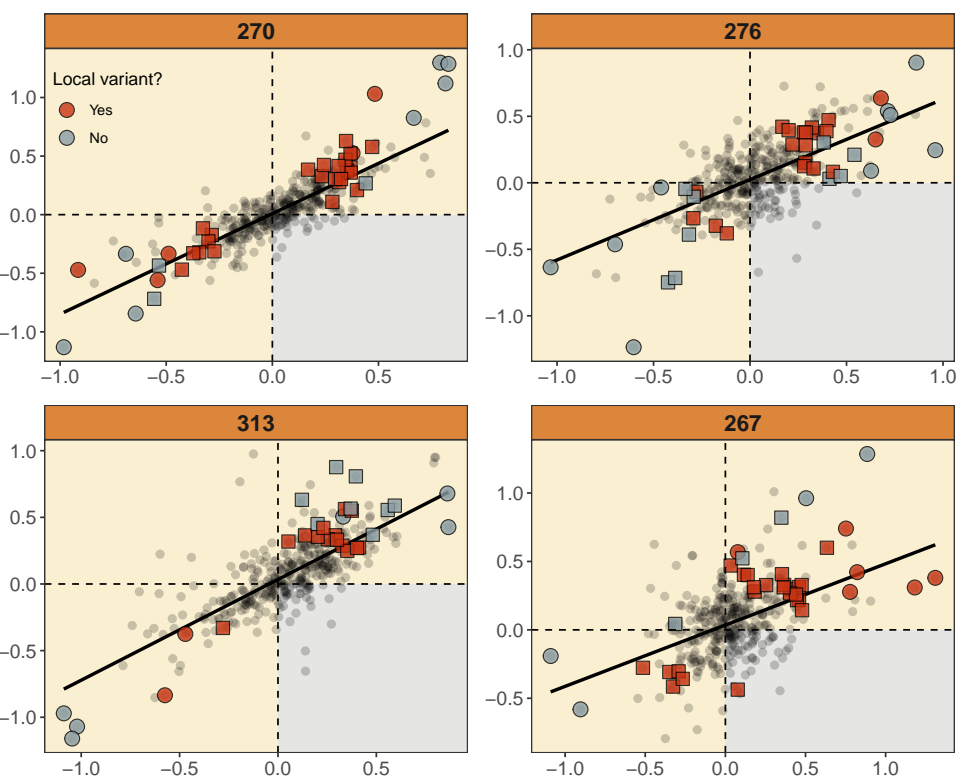

Beta score (nodule number)
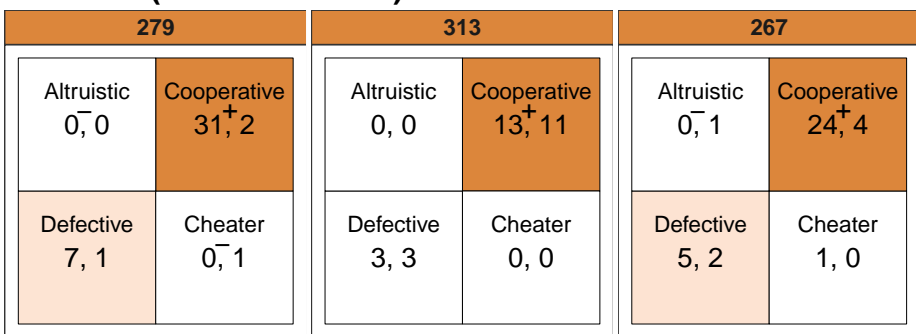

Figure 3: Most significant genomic variants were cooperative and local. A) GWAS beta scores for symbiont quality (shoot biomass) and symbiont fitness (nodule number) for the 363 variants (points) among the 40 derived isolates, averaged over all plant lines ('All') or for each host genotype separately (other panels). Larger symbols are variants with one or more statistically significant effects (segregating in the ancestor in squares, de novo in circles). Variants evolved in individual host environments (red) or not (grey), except in the 'All' panel (black). Grey region defines 'cheater' variants. (B) Counts of significant variants in each host environment. See text for category definitions. Numbers before the commas indicate local variants and numbers after commas indicates variants that evolved in a different host environment. + or - marks categories with more or fewer variants, respectively, than the null expectation.

on the host genotype with which they shared evolutionary history (Fig. 2), and we detected a significant excess of genomic variants with cooperative effects on local hosts (Fig. 3). These results suggest that local adaptation is a more important evolutionary force shaping microbial cooperation than partner choice. Furthermore, when microbes are consistently paired with the same host and dispersal is limited, the resulting local adaptation likely leads to more, not less, cooperation. What emerges is a model of adaptation in host-microbe mutualisms: microbes can rapidly adapt to a 
particular host genotype via standing or de novo variants that also benefit local hosts but have varying effects on non-local host genotypes, maintaining the variation in mutualist quality that we observe in host-associated microbiomes. Our results also imply that transplanting microbiota is more likely to be effective among closely related hosts, or that one needs to give sufficient time for microbes to adapt to a new host environment.

\section{References}

1. M. E. Frederickson, Mutualisms Are Not on the Verge of Breakdown. Trends in Ecology and Evolution 32, 727-734 (2017).

2. J. L. Sachs, C. J. Essenberg, M. M. Turcotte, New paradigms for the evolution of beneficial infections. Trends in Ecology and Evolution 26, 202-209 (2011).

3. K. D. Heath, J. R. Stinchcombe, Explaining mutualism variation: A new evolutionary paradox? Evolution 68, 309-317 (2014).

4. J. L. Sachs, M. O. Ehinger, E. L. Simms, Origins of cheating and loss of symbiosis in wild Bradyrhizobium. Journal of Evolutionary Biology 23, 1075-1089 (2010).

5. K. R. Foster, J. Schluter, K. Z. Coyte, S. Rakoff-Nahoum, The evolution of the host microbiome as an ecosystem on a leash. Nature 548, 43-51 (2017).

6. E. T. Kiers, R. A. Rousseau, S. A. West, R. F. Denison, Host sanctions and the legumerhizobium mutualism. Nature 425, 78-81 (2003).

7. K. D. Heath, P. Tiffin, Stabilizing mechanisms in a legume-rhizobium mutualism. Evolution 63, 652-662 (2009).

8. A. K. Simonsen, J. R. Stinchcombe, Standing genetic variation in host preference for mutualist microbial symbionts. Proceedings of the Royal Society B: Biological Sciences 281, 214-217 (2014).

9. R. T. Batstone, E. M. Dutton, D. Wang, M. Yang, M. E. Frederickson, The evolution of symbiont preference traits in the model legume Medicago truncatula. New Phytologist 213, 1850-1861 (2017).

10. K. R. Foster, H. Kokko, Cheating can stabilize cooperation in mutualisms. Proceedings of the Royal Society B: Biological Sciences 273, 2233-2239 (2006).

11. P. H. Thrall, J. Burdon, M. J. Woods, Variation in the effectiveness of symbiotic associations between native rhizobia and temperate Australian legumes: interactions within and between genera. Journal of Applied Ecology 37, 52-65 (2000).

12. M. A. Rúa et al., Home-field advantage? Evidence of local adaptation among plants, soil, and arbuscular mycorrhizal fungi through meta-analysis. BMC Evolutionary Biology 16, 115 (2016).

13. K. D. Heath, Intergenomic epistasis and coevolutionary constraint in plants and rhizobia. Evolution 64, 1446-1458 (2010). 
14. J. D. Hoeksema, Ongoing coevolution in mycorrhizal interactions. New Phytologist 187, 286-300 (2010).

15. S. S. Porter, M. L. Stanton, K. J. Rice, Mutualism and adaptive divergence: Co-invasion of a heterogeneous grassland by an exotic legume-rhizobium symbiosis. PLOS ONE 6 (2011).

16. T. L. Harrison, C. W. Wood, I. L. Borges, J. R. Stinchcombe, No evidence for adaptation to local rhizobial mutualists in the legume Medicago lupulina. Ecology and Evolution 7, 43674376 (2017).

17. P. Remigi, J. Zhu, J. P. W. Young, C. Masson-Boivin, Symbiosis within Symbiosis: Evolving Nitrogen-Fixing Legume Symbionts. Trends in Microbiology 24, 63-75 (2016).

18. B. Epstein et al., Genome-wide association analyses in the model Rhizobium Ensifer meliloti. MSphere 3, e00386-18 (2018).

19. L. T. Burghardt et al., Select and resequence reveals relative fitness of bacteria in symbiotic and free-living environments. Proceedings of the National Academy of Sciences 115, 24252430 (2018).

20. Materials and methods are available as supporting material on Science Online.

21. F. Galibert et al., The composite genome of the legume symbiont Sinorhizobium meliloti. Science 293, 668-672 (2001).

22. T. M. Finan et al., The complete sequence of the 1,683-kb pSymB megaplasmid from the N2-fixing endosymbiont Sinorhizobium meliloti. Proceedings of the National Academy of Sciences 98, 9889-9894 (2001).

23. K. M. Jones, H. Kobayashi, B. W. Davies, M. E. Taga, G. C. Walker, How rhizobial symbionts invade plants: the Sinorhizobium-Medicago model. Nature Reviews Microbiology 5, 619-633 (2007).

24. R. F. Denison, Legume Sanctions and the Evolution of Symbiotic Cooperation by Rhizobia. The American Naturalist 156, 567-576 (2000).

25. S. A. West, E. T. Kiers, E. L. Simms, R. F. Denison, Sanctions and mutualism stability: Why do rhizobia fix nitrogen? Proceedings of the Royal Society B: Biological Sciences 269, 685694 (2002).

26. R. Oono, C. G. Anderson, R. F. Denison, Failure to fix nitrogen by non-reproductive symbiotic rhizobia triggers host sanctions that reduce fitness of their reproductive clonemates. Proceedings of the Royal Society B: Biological Sciences 278, 2698-2703 (2011).

27. E. T. Kiers, W. C. Ratcliff, R. F. Denison, et al., Single-strain inoculation may create spurious correlations between legume fitness and rhizobial fitness. New Phytologist 198, 4-6 (2013).

28. M. L. Friesen, K. D. Heath, et al., One hundred years of solitude: integrating single-strain inoculations with community perspectives in the legume-rhizobium symbiosis. New Phytologist 198, 7-9 (2013).

29. K. A. Gano-Cohen et al., Interspecific conflict and the evolution of ineffective rhizobia. Ecology Letters 22, 914-924 (2019).

30. M. E. Frederickson, No selection for cheating in a natural meta-population of rhizobia. Ecology Letters 23, 409-411 (2020). 
31. S. Rome, B. Brunel, P. Normand, M. Fernandez, J. C. Cleyet-Marel, Evidence that two genomic species of Rhizobium are associated with Medicago truncatula. Archives of Microbiology 165, 285-288 (1996).

32. J. J. Terpolilli, G. W. O’Hara, R. P. Tiwari, M. J. Dilworth, J. G. Howieson, The model legume Medicago truncatula A17 is poorly matched for $\mathrm{N}^{2}$ fixation with the sequenced microsymbiont Sinorhizobium meliloti 1021. New Phytologist 179, 62-66 (2008).

33. D. G. Barker et al., Growing M. truncatula: choice of substrates and growth conditions, Ardmore, OK, US, 2006, (http://www.noble.org/MedicagoHandbook).

34. K. D. Heath, A. J. Stock, J. R. Stinchcombe, Mutualism variation in the nodulation response to nitrate. Journal of Evolutionary Biology 23, 2494-2500 (2010).

35. M. A. Grillo, J. R. Stinchcombe, K. D. Heath, Nitrogen addition does not influence preinfection partner choice in the legume-rhizobium symbiosis. American Journal of Botany 103, 1763-1770 (2016).

36. P. Somasegaran, H. J. Hoben, Handbook for rhizobia: methods in legume-Rhizobium technology (Springer Science \& Business Media, 2012).

37. M. L. Friesen, Widespread fitness alignment in the legume-rhizobium symbiosis. New Phytologist 194, 1096-1111 (2012).

38. M. L. Friesen et al., The ecological genomic basis of salinity adaptation in Tunisian Medicago truncatula. BMC Genomics 15 (2014).

39. D. Bates, M. Maechler, B. Bolker, S. Walker, Fitting Linear Mixed-Effects Models Using lme4. Journal of Statistical Software 67, 1-48, (https://cran.r-project.org/web/packages/ lme4/lme4.pdf) (2015).

40. J. Fox, S. Weisberg, An $R$ companion to applied regression (Sage publications, Thousand Oaks, CA, US, 2018).

41. D. Herr, On the history of ANOVA in unbalanced, factorial-designs. The American Statistician 40, 265-270 (1986).

42. Ø. Langsrud, ANOVA for unbalanced data: Use Type II instead of Type III sums of squares. Statistics and Computing 13, 163-167 (2003).

43. M. L. Delignette-Muller, C. Dutang, fitdistrplus: An R Package for Fitting Distributions. Journal of Statistical Software 64, (https://cran.r-project.org/web/packages/fitdistrplus/ fitdistrplus.pdf) (2015).

44. F. Hartig, DHARMa: Residual Diagnostics for Hierarchical (Multi-Level / Mixed) Regression Models. 2018, (https://cran.r-project.org/package=DHARMa).

45. X. Zhang, L. Wang, J. Li, R. T. Batstone, M. E. Frederickson, Medicago truncatula adjusts root proliferation, nodule formation, and partner choice in response to local $\mathrm{N}$ heterogeneity. Plant and Soil 450, 417-428 (2020).

46. W. Taiyun, S. Viliam, $R$ package 'corrplot': Visualization of a Correlation Matrix, 2017, (https://github.com/taiyun/corrplot).

47. S. Via, R. Lande, Genotype-Environment Interaction and the Evolution of Phenotypic Plasticity. Trends in Ecology and Evolution 39, 505-522 (1985). 
48. R. L. Trivers, The evolution of reciprocal altruism. The Quarterly Review of Biology 46, 3557 (1971).

49. R. Axelrod, W. D. Hamilton, The evolution of cooperation. Science 211, 1390-1396 (1981).

50. H. Li, Seqtk Toolkit for processing sequences in FASTA/Q formats, 2012, (https://github.com/ lh3/seqtk).

51. H. Li, Aligning sequence reads, clone sequences and assembly contigs with BWA-MEM (arXiv:1303.3997), 2013, (https://arxiv.org/abs/1303.3997).

52. A. Picard, Set of command line tools (in Java) for manipulating high-throughput sequencing (HTS) data and formats such as SAM/BAM/CRAM and VCF, 2018, (http://broadinstitute. github.io/picard/).

53. G. A. Van der Auwera et al., From FastQ data to high-confidence variant calls: the genome analysis toolkit best practices pipeline. Current Protocols in Bioinformatics 43, 11-10 (2013).

54. P. Danecek et al., The variant call format and VCFtools. Bioinformatics 27, 2156-2158 (2011).

55. J. R. Conway, A. Lex, N. Gehlenborg, UpSetR: an R package for the visualization of intersecting sets and their properties. Bioinformatics 33, 2938-2940 (2017).

56. T. L. Harrison, C. W. Wood, K. D. Heath, J. R. Stinchcombe, Geographically structured genetic variation in the Medicago lupulina-Ensifer mutualism. Evolution 71, 1787-1801 (2017).

57. M. Nei, Molecular Evolutionary Genetics (Columbia University Press, 1987).

58. X. Zhou, M. Stephens, Efficient multivariate linear mixed model algorithms for genome-wide association studies. Nature Methods 11, 407 (2014).

59. R Core Team, $R$ : A language and environment for statistical computing, 2016, (https://www. r-project.org/).

\section{Acknowledgements}

We thank volunteers J. Li, C. Chen, G. Forguson, F. Samad-zada, L. Wang, X. Zhang, D. Li, K. Ong, and J. Wong. The Frederickson Lab, especially E. Dutton and J. Laurich, as well as L. Burghardt, J. Lau, J. Stinchcombe, and C. Wood, provided feedback. I. Anreiter assisted with qPCR, and A. Petrie, B. Cole, and C. Bonner managed the greenhouse. Funding: We acknowledge funding from an NSERC Discovery Grant and Accelerator Supplement (M.E.F.), Ontario Graduate (R.T.B.) and NSERC (T.L.H.) Scholarships, and the University of Toronto. Author contributions: R.T.B. and M.E.F. conceived the study and designed experiments; R.T.B. conducted experiments; R.T.B. and M.E.F. analyzed phenotypic data; R.T.B., A.M.O. and T.L.H. conducted bioinformatics; all authors wrote the manuscript. Competing interests: Authors declare no competing interests. 
Data and materials availability: Raw sequence reads were deposited to NCBI at BioProject ID PRJNA512862. All data and code for analyses are available at this permanent repository. 


\title{
Supplementary materials
}

\author{
Materials and Methods \\ Figs. S1 to S6 \\ Tables S1 to S4
}

\section{Materials and methods}

Study system Legume-rhizobium interactions are often used to study the evolution of cooperation between species, or mutualism (e.g., 4, 6, 7, 9). Legumes host rhizobia in root nodules, where they exchange plant-fixed carbon for rhizobium-fixed nitrogen $(6,9)$. Nitrogen fixation by rhizobia is energetically costly, leading many authors to expect rhizobia to 'cheat' by failing to fix nitrogen (24-26). However, how much fitness conflict actually occurs between legumes and rhizobia is hotly debated (27-30), meaning there is little consensus about whether rhizobia adapt to hosts by becoming more or less beneficial.

Here, we studied Medicago truncatula, an annual legume native to the Mediterranean region. It forms indeterminate nodules and typically associates with rhizobia in the genus Ensifer (31). Medicago truncatula seeds were obtained from the Medicago Hapmap Project. We experimentally evolved E. meliloti strains 1021 and 1022 (hereafter Em1021 and Em1022) with five inbred lines of M. truncatula that vary in their preference for Em1022. We chose the five plant lines to span the variation in partner choice we measured in a previous study (9). Line 270 is not choosy; it forms similar numbers of nodules with both strains. Line 267 forms almost all of its nodules with Em1022. The other three lines $(276,279$, and 313) are intermediate, preferring Em1022 to Em1021 to varying degrees. In all figures, plant lines are ordered by increasing preference for Em1022.

Inbred lines HM270, HM276, HM279, HM313, and HM267 were originally collected from distinct regions in Portugal, Algeria, Morocco, Canada, and Algeria, respectively. Em1021 is the canonical rhizobium strain and was the first to have its entire genome sequenced $(21,22)$. Em1022 was originally isolated from the annual legume Medicago orbicularis (J. G. Howieson, CRS, WSM GeneBank) growing under field conditions in Naxos, Greece. Isolates of Em1022 were provided by Jason Terpolilli from Murdoch University, Australia and isolates of Em1021 were provided by 
Turlough Finan from McMaster University, Canada. Em1022 fixes around three times the amount of nitrogen $(\mathrm{N})$ than the ineffective strain Em1021 (32), making it a higher-quality partner from the plant's perspective. In a study in which Em1021 and Em1022 were singly-inoculated on to 35 Medicago truncatula genotypes, Em1022 made plants grow significantly larger overall, but with significant $\mathrm{G} \times \mathrm{G}$ interactions (9).

Evolution experiment Initially, 55 replicates of each plant line were prepared according to published protocols (33). Seeds that germinated were planted in autoclaved Magenta boxes (34, 35). Briefly, seeds were nick-scarified, submerged in $95 \%$ ethanol for $30 \mathrm{~s}$ and then $6 \%$ bleach for 4 min, rinsed under distilled water for $5 \mathrm{~min}$, and imbibed in distilled water for $15 \mathrm{~min}$. Swollen seeds were placed on autoclaved filter paper covering water agar, sealed, stored at $4{ }^{\circ} \mathrm{C}$ in the dark for $2 \mathrm{~d}$, and at room temperature for an additional day. A single seedling was planted into a Magenta Box consisting of two compartments stacked vertically; the top compartment was filled with autoclaved washed river sand (New Canadian Lumber and Building Supplies, Toronto, Canada), while the bottom compartment was filled with autoclaved $\frac{1}{4}$-strength $\mathrm{N}$-free Fahräeus medium (33) to provide plants with essential nutrients other than nitrogen. An autoclaved polypropylene wick (Blueline, $\frac{3}{8}$-in diameter rope) spanned the two compartments and wicked fertilizer into the sand; fertilizer in bottom compartments was replenished weekly.

Plants were randomly distributed across three benches of a greenhouse room at the University of Toronto, Canada, from April 2016 until April 2017, with temperature set to $23^{\circ} \mathrm{C}$ with supplemental 16h day, $8 \mathrm{hr}$ night lighting. Three days after planting, when most seedlings had developed their first true leaves, we inoculated each plant with $1 \mathrm{ml}$ of a 2:1 mixture of Em1021:Em1022, each strain having been cultured separately in liquid Tryptone Yeast (TY) media (36) for $3 \mathrm{~d}$ in a shaking incubator at $29^{\circ} \mathrm{C}$ and $200 \mathrm{rpm}$; both cultures reached optical densities of $0.1-0.15$, or $c$. $10^{7}$ cells per ml. We started the experiment with twice the number of Em1021 than Em1022 cells because we expected choosy plant lines to strongly favor Em1022 and we did not want Em1021 to go extinct in a single generation. 
Plants grew for 5-6 weeks and then nodules senesced in soil for 3 weeks, meaning two months elapsed between plant generations. We planted one seed from the same plant line back into each pot. We repeated this process four more times for a total of five plant generations. All methods were identical across generations, except plants were inoculated with rhizobia only in the first generation; subsequently, only rhizobia present in the soil that survived the previous generation(s) could nodulate plants. At the end of each generation (after $\sim 6$ wks of growth), we harvested plants by carefully removing roots from sand and severing the shoot from the roots. We counted all nodules, and then weighed aboveground plant parts to the nearest $0.001 \mathrm{mg}$ after drying in an oven set to $60^{\circ} \mathrm{C}$ for two days. We then re-planted the roots and nodules back in the same pot and sand, giving nodules an additional three weeks to senesce and release rhizobia into the sand prior to each pot receiving a new seed from the same plant line. In other words, rhizobia in each pot were paired with the same plant line throughout the entire evolution experiment, and plant growth and harvest conditions remained the same. Although only a single plant line was present in each pot, competition among rhizobia strains was possible (e.g., between Em1021 and Em1022 at the beginning of the experiment, and among isolates of Em1022 throughout the experiment). At the end of the experiment, in addition to measuring nodule number and shoot biomass, we also sub-sampled nodules and isolated their rhizobia to determine strain identity and estimated strain abundance in the soil using quantitative PCR (see below). In total, we harvested 1,178 plants throughout the evolution experiment, an average of 36 plants per line per generation (range: 11-55) (Fig. S6).

Quantifying bacterial relative abundance At the end of plant generations 2-4 of the evolution experiment, we tracked rhizobial strain abundance in the soil by destructively harvesting five haphazardly chosen replicates per line and conducting quantitative PCR (qPCR). To prepare soil samples for DNA extraction, we weighed $50 \mathrm{~g}$ of sand from each pot, added $20 \mathrm{ml} 0.85 \% \mathrm{NaCl}$ solution, vortexed for $20 \mathrm{~s}$, and pipetted off $5 \mathrm{ml}$ of the cloudy liquid, putting $1 \mathrm{ml}$ into each of five microcentrifuge tubes. Tubes were centrifuged for two mins at 15,000 x g, and supernatants were 
poured off. The resulting five pellets from each sample were then recombined using the buffer solution from step 1 of the DNA extraction protocol of the E.Z.N.A. Soil DNA Kit (Omega Bio-tek, Georgia, US), and all downstream steps were completed as per the protocol. We designed custom ®TaqMan Gene Expression Assays (Thermo Fisher Scientific, Carlsbad, CA, US) based on the nodC locus (Table S4) because it was sufficiently divergent between Em1021 and Em1022 to discriminate between strains. For every sample, we completed three technical replicates; we analyze data only from samples with technical replicates within 0.2 standard deviations of one another. In every qPCR run, we included a standard curve for both Em1021 and Em1022, whereby the initial concentration of each sample was known. We could thus calculate the proportion of Em1022 in each sample by dividing the starting quantity of DNA belonging to Em1022 by total DNA starting quantity $($ Em1022 + Em1021).

Obtaining derived isolates At the end of the evolution experiment, we dissected nodules from each plant, and stored dissected nodules atop cotton and silica gel in microcentrifuge tubes at $4{ }^{\circ} \mathrm{C}$. Prior to surface sterilization and plating, we transferred nodules to tubes filled with distilled water to imbibe them overnight. We surface-sterilized nodules using 95\% ethanol (15 s), $6 \%$ hypochlorite (15 s), and five changes of distilled water, and then crushed each individual nodule and streaked its contents onto a solid TY media plate. After growing for three days in an incubator set to $29^{\circ} \mathrm{C}$, we transferred a loopful of bacteria into liquid TY media that was placed in a shaking incubator set to $29^{\circ} \mathrm{C}$ and $200 \mathrm{rpm}$. After three days, $1 \mathrm{ml}$ of each culture was transferred to a cryotube containing autoclaved glycerol (15\% final concentration), flash frozen in liquid nitrogen, and stored at $-80^{\circ} \mathrm{C}$.

To distinguish between Em1021 and Em1022 in nodules at the end of the experiment, we used selective antibiotic media; only Em1021, and not Em1022, is resistant to streptomycin (9). For every cultured isolate, we streaked two plates, one with and the other without streptomycin (100 $\mu \mathrm{g}$ $\mathrm{ml}^{-1}$ ). Bacteria that grew on both plates were scored as Em1021; bacteria that grew only on plates without streptomycin were scored as Em1022. We could thus calculate the proportion of Em1022 formed by each plant by counting the number of nodules scored as Em1022 and dividing by total 
nodules scored per plant. We also tested for streptomycin resistance in our derived isolates, which were all confirmed to be Em1022 based on whole-genome sequencing, and found no evidence that the evolution experiment gave rise to streptomycin resistance in strain Em1022.

Single-strain inoculations of ancestral and derived rhizobia We haphazardly chose 40 derived isolates resulting in 12 isolates from plant line 270, 3 from plant line 276, 11 from plant line 279, 6 from plant line 313, and 8 from plant line 267. These isolates were all Em1022; they were all susceptible to streptomycin (Em1021 is resistant) and closely related to ancestral Em1022 based on whole-genome sequencing. We obtained unequal numbers of isolates from each plant line because we chose isolates in proportion to how many nodules plant lines formed at the end of the experiment.

We inoculated each isolate singly onto five replicates of each of the same five plant lines (5 replicates x 5 plant lines $\times 40$ derived isolates $=1,000$ plants, for a total of $200 \mathrm{G} \times \mathrm{G}$ combinations). Because of the size of this experiment, we initiated a new batch of seeds every day July 3-7 and July 17-21, 2017. For each batch, we always included ancestral Em1022 (3 replicates per plant line) together with four randomly chosen derived isolates. We prepared seeds of plant lines 270, 276, 279,313 , and 267 using the same protocols as in the evolution experiment (above), and planted a single germinated seed into an autoclaved Ray-Leach Recycled SC10 Cone-tainer ${ }^{\mathrm{TM}}$ assembled much like our Magenta boxes (see above). Every day, we also included 2-3 un-inoculated plants per line to assess contamination. Cone-tainers ${ }^{\mathrm{TM}}$ were filled with washed river sand, and their bottoms plugged with a clean polyester cotton ball to prevent sand from falling out. The bottom of each Cone-tainer ${ }^{\mathrm{TM}}$ was fastened to a sterile $50 \mathrm{ml}$ Falcon tube (Corning Life Sciences) filled with the same $\mathrm{N}$-free fertilizer as used in the evolution experiment. We then used a polypropylene wick to span from two inches below the surface of the sand to the very bottom of the Falcon tube, such that the sand remained moist throughout the experiment. Assembled Cone-tainers ${ }^{\mathrm{TM}}$ were autoclaved twice to completely sterilize all components prior to planting.

All plants were placed in a random position in trays covered with a transparent bag to retain 
humidity. Trays were kept in a growth chamber set to $23^{\circ} \mathrm{C} / 18^{\circ} \mathrm{C}$ day/night temperature, $16 \mathrm{~h} / 8 \mathrm{~h}$ light/dark photoperiod, and $300 \mu \mathrm{mol} \mathrm{m} \mathrm{s}^{-1}$ photosynthetically active radiation (PAR), and rotated frequently around the growth chamber. At the end of the experiment (after $\sim 4$ wks growth), we quantified the symbiotic quality of each rhizobium isolate by measuring the aboveground shoot biomass of its host plant, a measure that is commonly used as a proxy for rhizobia quality as it reflects how much nitrogen rhizobia fix for their hosts $(37,38)$. We dried shoots for two days in an oven at $60^{\circ} \mathrm{C}$, and then weighed them to the nearest $0.001 \mathrm{mg}$. We used nodule number as a measure of the fitness of a rhizobia strain when singly inoculated on a plant (13). Contamination was minimal: only a single control plant of 66 that survived until the end of the experiment was found to produce three small, white nodules. In total, we harvested 1,107 plants at the end of the single-strain inoculation experiment, an average of five plants per line per isolate (range: 3-5).

Statistical analyses All code for the following analyses is available at this permanent repository. We used a linear or generalized linear mixed model (G)LMM framework in R using the lme4 package (39). In the single inoculation experiment, we included isolate and tray as random effects. We tested the significance of main and interaction effects using Wald Chi-squared tests calculated in the car package in R (40) with type III sums of squares, or type II sums of squares when interaction terms were not significant $(41,42)$. We used the fitdist package in $\mathrm{R}(43)$ to select the best-fitting (i.e., lowest AIC value) probability distribution for each trait (Tables S1 \& S2). For GLMMs, we checked model fit and assessed scaled residuals using the DHARMa package in R v $0.1 .6(44)$.

Does the proportion of the higher-quality strain change over time? Using qPCR and nodule occupancy data, we first determined how the proportion of Em1022 changed over the course of the evolution experiment using a linear model that included the main and interactive fixed effects of plant line (five levels: 270, 276, 279, 313, 267) and the plant generations for which data were available (four ordered levels, generations 2-5). A priori, we expected that both partner choice by hosts and competition among strains might influence rhizobia evolution in our experiment. Although 
we did not directly test the competitive abilities of Em1021 versus Em1022 in the present study, in previous work, plants formed $\sim 40 \%$ more nodules with Em1022 when Em1021 and Em1022 were spatially separated (via a split-root setup) on the same root system (9), but plants formed $\sim 80 \%$ more nodules with Em1022 when root halves received both Em1021 and Em1022 and the strains could directly interact (45). In other words, Em1022 had greater relative abundance than Em1021 when the two strains could directly interact, compared to when the two strains were spatially separated. This difference suggests that strain-strain interactions likely play an important role in determining strain frequencies in plants. Similarly, Burghardt et al. (19) found that E. meliloti strain fitness was only weakly correlated in single versus mixed inoculations, further evidence that direct interactions between strains modulate strain success.

Do measures of rhizobia quality and fitness differ between ancestral and derived states? For the single inoculation experiment, we first tested whether derived Em1022 isolates evolved to be better or worst mutualists overall, compared to the ancestral Em1022. We conducted LMMs and GLMMs for measures of symbiont quality (shoot biomass) and fitness (nodule number), respectively, that included the main and interactive effects of bacterial treatment (two levels: ancestral, derived), and assayed plant line (five levels: 270, 276, 279, 313, 267). We also included tray and isolate as random effects.

Do rhizobia quality and fitness depend on whether they share an evolutionary history with their host? We quantified whether symbiont quality and fitness depended on whether isolates shared an evolutionary history with the tested plant line using (G)LMMs that included the main and interactive fixed effects of shared evolutionary history (2 levels: yes, no) and assayed plant line (five levels: 270, 276, 279, 313, 267). We also included tray and isolate as random effects.

Does rhizobia quality or fitness trade off across host lines? We calculated raw isolate means within each host line, thus generating an estimate for every isolate-line combination $(\mathrm{N}=40$ isolates x 5 lines $=200$ combinations). Using the corrplot package in v.0.84 (46), we calculated Pearson correlation coefficients between isolate means on one assayed plant line and isolate means on another plant line for all pairwise combinations of plant lines. Significant positive correlations 
indicate that isolate quality on one assayed plant line is similar to its quality on another, while negative correlations would indicate isolate quality on one line trades off with its quality on another (47). To facilitate among-line comparisons of each isolate, we standardized isolate trait values by subtracting the line mean from each and dividing by the line standard deviation.

Are legume and rhizobium fitness aligned? Models predict that mutualists should be under selection to 'cheat', i.e., gain fitness at their partner's expense $(48,49)$. However, empirical work on the legume-rhizobium mutualism in particular has instead shown widespread fitness alignment, whereby greater cooperation is associated with higher fitness in both interacting species $(28,37)$. Using the isolate means averaged across plant lines, we regressed shoot biomass against nodule number in a univariate linear model to test whether derived isolates evolved to 'cheat', i.e., experience a gain in fitness (nodule number) at the plant's expense (shoot biomass). Cheaters would thus fall in the bottom right quadrant of Figure S3, yet not a single isolate is significantly within this quadrant. Similarly, at the genomic-level, only 1 of the 145 significant variants is (very weakly) positively associated with nodule number but negatively associated with shoot biomass (Fig. 3A), and there are significantly fewer 'cheater' variants than we would expect by chance, given our randomization results, further suggesting a paucity of derived 'cheaters.'

Whole-genome sequencing We extracted DNA from the 40 derived and nine ancestral replicates of Em1022 using the GenElute ${ }^{\mathrm{TM}}$ Bacterial Genomic DNA isolation kit (Sigma-Aldrich) and submitted extractions to a sequencing facility (Centre for Analysis of Genome Evolution and Function, CAGEF, at the University of Toronto) for library preparation and sequencing. The nine ancestral Em1022 replicates were obtained via re-streaking and culturing the original Em1022 isolate used at the beginning of the evolution experiment. Illumina libraries were prepared using the Nextera XT DNA Library preparation kit (Illumina) according to the manufacturer's guidelines. Libraries were dual barcoded using the Nextera XT Index Kit, pooled in equal amounts, and then denatured and diluted to a sequencing concentration of $1.8 \mathrm{pM}$. Sequencing was performed on an Illumina NextSeq 500 using the Mid Output V2 sequencing kit with $150 \times 2$ paired-end reads to an average 
read depth of $\sim 100 x$.

Variant discovery We first used seqtk (50) to trim the low-quality ends of the raw reads. We then aligned both the forward and reverse reads using bwa (51) to the reference genome of Ensifer meliloti strain KH35c (Assembly ID: GCA_002197105.1; replicon IDs: chromosome CP021825.1, accessory plasmid A CP021826.1, symbiosis plasmid A CP021827.1, and symbiosis plasmid B CP021828.1) using default parameters. We chose the strain Ensifer meliloti $\mathrm{KH} 35 \mathrm{c}$ as the reference because it is the closest relative to Em1022 that has been fully Pac-Bio sequenced. We used PICARD tools (52) to reorder, add read groups, sort, and mark duplicates for each alignment. We then used GATK's Best Practices (53) for variant discovery, first taking each individual sequence and calling all SNPs and indels that differed from the reference sequence using Haplotype Caller, and then taking all sequences together and calling SNPs and indels using the joint genotyping tool, genotypeGVCF. We used hard-filtering options in vcftools (54) to include only variants with a minimum read depth (DP) of 20, maximum DP of 230, maximum missing data of $10 \%$, minimum genotype quality of 30, and excluded sites that were invariant among our samples but different from the reference strain. These filters reduced our SNP set from 21,247 to 1330 genomic variants.

We then used the $\mathrm{R}$ package UpSetR (55) to visualise the number of unique variants that evolved on a particular host and shared variants among hosts (Fig. S5). We checked whether the number of unique variants was correlated with the overall number of isolates sequenced from each host, and did not find any discernible pattern. For example, we sequenced similar numbers of isolates for lines $279(\mathrm{~N}=11)$ and $270(\mathrm{~N}=12)$ but found five-times fewer unique variants on line 279 (59 vs 305, respectively). Using a custom python script (56), we calculated the average pairwise nucleotide differences $(\pi)(57)$ among derived and ancestral isolates, but found similar values (derived $\pi=0.216$, ancestral $\pi=0.211$; both per $10 \mathrm{kbps}$ ), suggesting that genetic variation among derived isolates was maintained throughout our evolution experiment, despite selection on rhizobia. This is perhaps not surprising, given that some new mutations arose that did not have time to fix, and relaxed selection may have allowed neutral variants to accumulate in their local 
host environment (some of which may have been deleterious on non-local hosts).

GWAS Given that the success of genome-wide association studies (GWAS) relies on statistically distinguishable variants, we removed most singletons and sites in high linkage disequilibrium (LD). Specifically, we identified all singleton variants, chose a single representative for each isolate with the highest quality, and excluded all others because singletons from the same isolate are in perfect LD. Based on the resulting 886 SNPs, we calculated the pairwise $r^{2}$ value that measures the degree to which variants are in LD with one another, grouped variants that were highly $\left(\mathrm{r}^{2}>0.95\right)$ linked, and chose the variant with the highest minor allele frequency and quality. The resulting 363 variants (Fig. S5) were used in a GWAS that tests for statistical associations between genomic variants and phenotypes (rhizobia quality or fitness). We used the program GEMMA (v0.98.1) (58), implementing the linear mixed model option (-1mm 4) with the standardized kinship matrix included as a random effect. We performed a GWAS for each isolate phenotype averaged across all plant hosts and for each isolate phenotype measured on each plant host individually, leading to six GWAS being run for rhizobial quality and six for rhizobial fitness.

GWAS has a number of well known statistical artefacts: it may erroneously assign effects to rare SNPs, especially if individuals carrying rare alleles also display extreme phenotypic values, even if the trait is controlled by more small effect alleles (18). Furthermore, the multiple testing nature inherent in the analysis will sometimes detect significance even when there is no association between genotype and phenotype. To address these issues, we created 1,000 randomly permuted phenotype datasets, which we linked with non-permuted sequencing information, i.e. we randomized phenotypes across genotypic values. We ran each permutation through the same GWAS pipeline, and recorded beta values (i.e., slopes of associations between phenotypes and genomic variants). We then summarized the distribution of beta values for each variant across all 1,000 permutations, which we used to calculate cutoff ranges of beta values for each variant, i.e. those exceeding the $2.5 \%$ and $97.5 \%$ intervals of this null distribution. To verify these values, we held each permuted dataset out in turn, and calculated the number of variants that would have been 
detected as significant if only the remaining permutations had built the null distribution. Averaged across all variants and permutations, this calculates the false discovery rate (FDR), and it matched the expected value of $5 \%$. Our permutations thus account for biases produced by the nature of our true allelic frequency distribution and trait data (18). We performed this analysis in $\mathrm{R}(59)$. 


\section{Supplementary Figures}

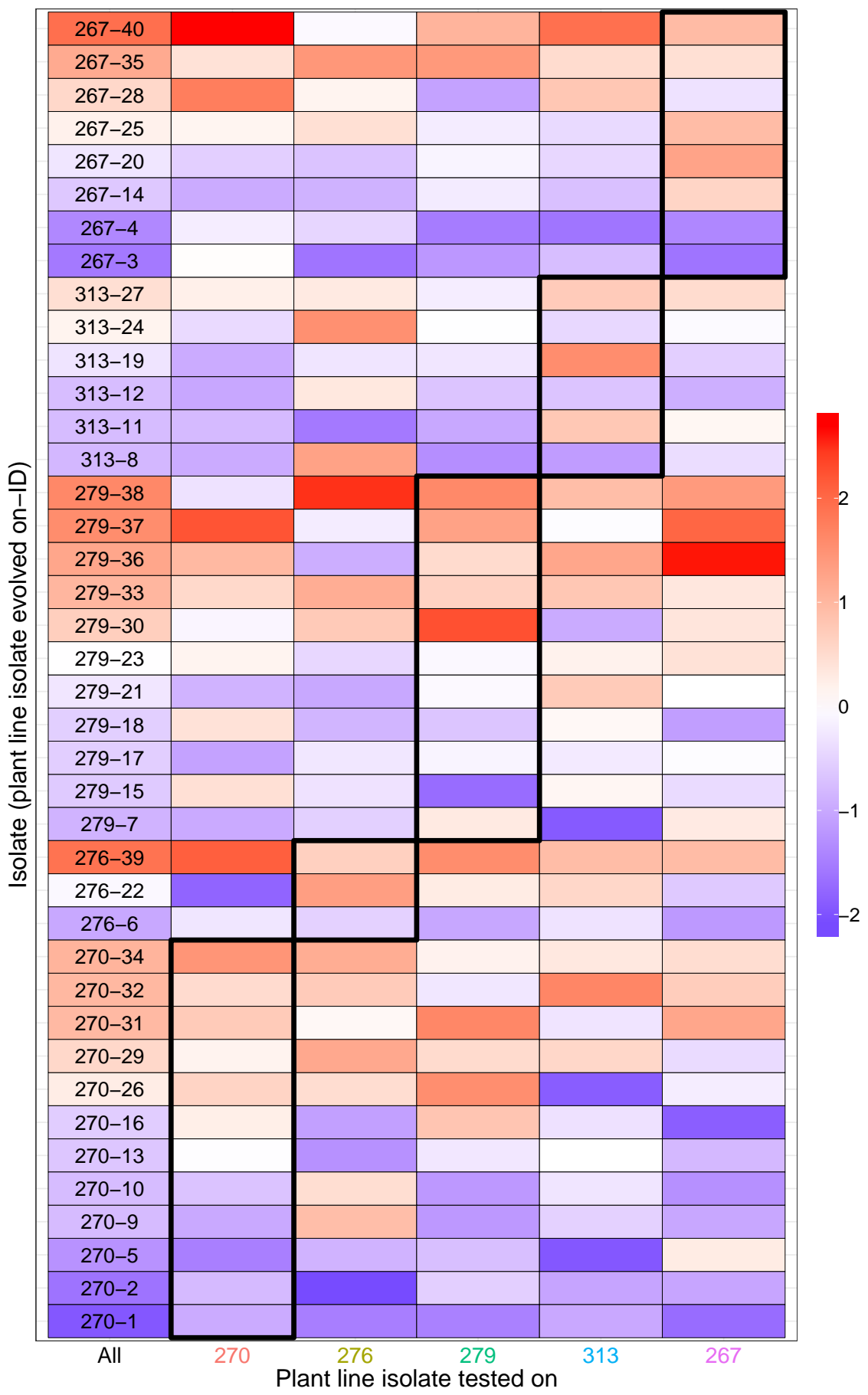

Figure S1: Heatmap showing the quality of every unique isolate when tested on each plant line. Isolate quality is the standardized raw mean for shoot biomass: (isolate mean - line mean)/line SD. In other words, red squares indicate isolates with higher than average quality on a host line, and blue squares indicate isolates with lower than average quality on a host line; comparisons are valid vertically (within a column), not horizontally (within a row isolates are standardized to different line means). The first column on the left shows average isolate effects across all plant lines, with unique isolate IDs after the dash. Boxes outlined in black show results for isolates tested on their local host. 


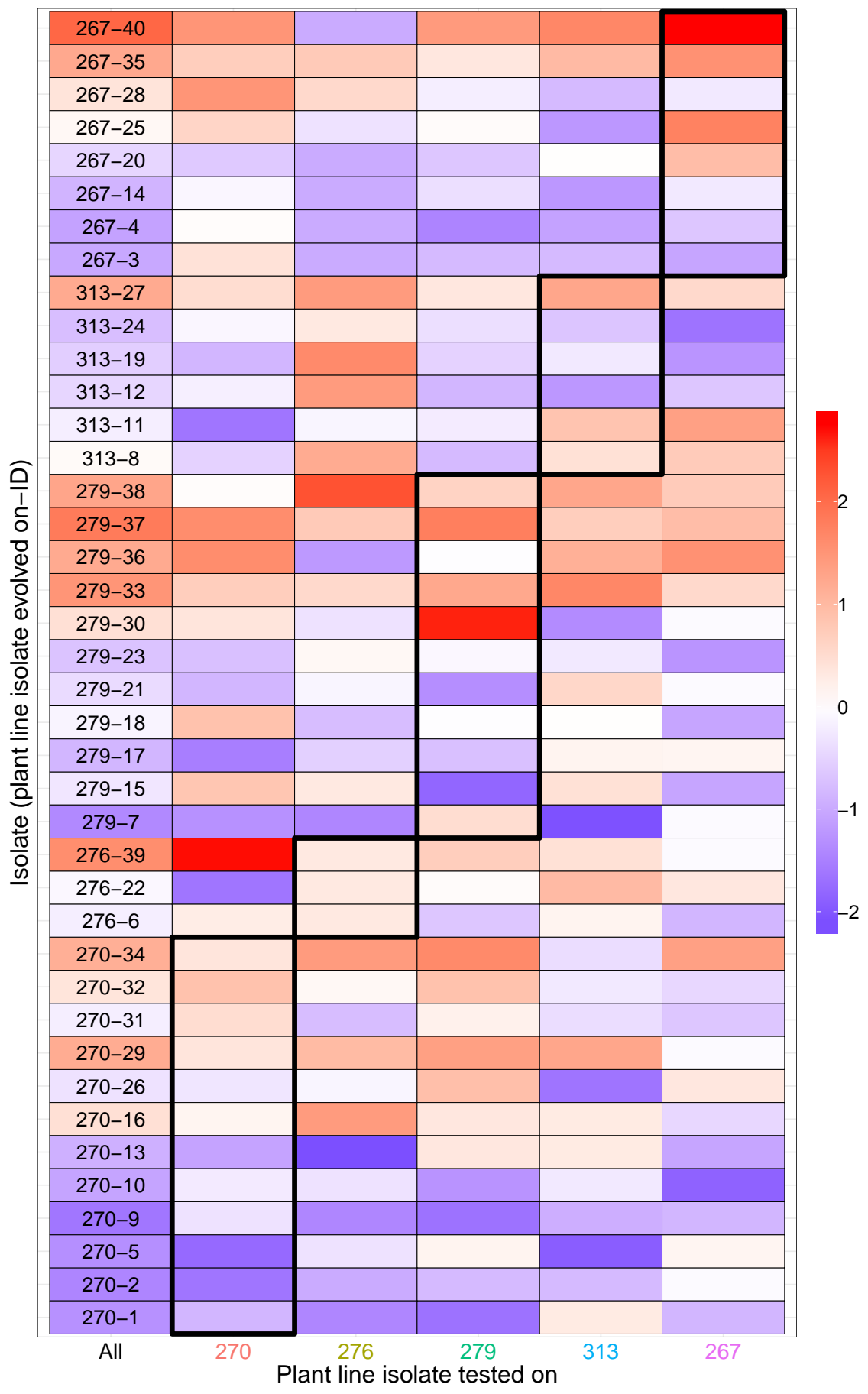

Figure S2: Heatmap showing the fitness of every unique isolate when tested on each plant line. Isolate fitness is the standardized raw mean for nodule number: (isolate mean - line mean)/line SD. In other words, red squares indicate isolates with higher than average fitness on a host line, and blue squares indicate isolates with lower than average fitness on a host line; comparisons are valid vertically (within a column), not horizontally (within a row isolates are standardized to different line means). The first column on the left shows average isolate effects across all plant lines, with unique isolate IDs after the dash. Boxes outlined in black show results for isolates tested on their local host. 


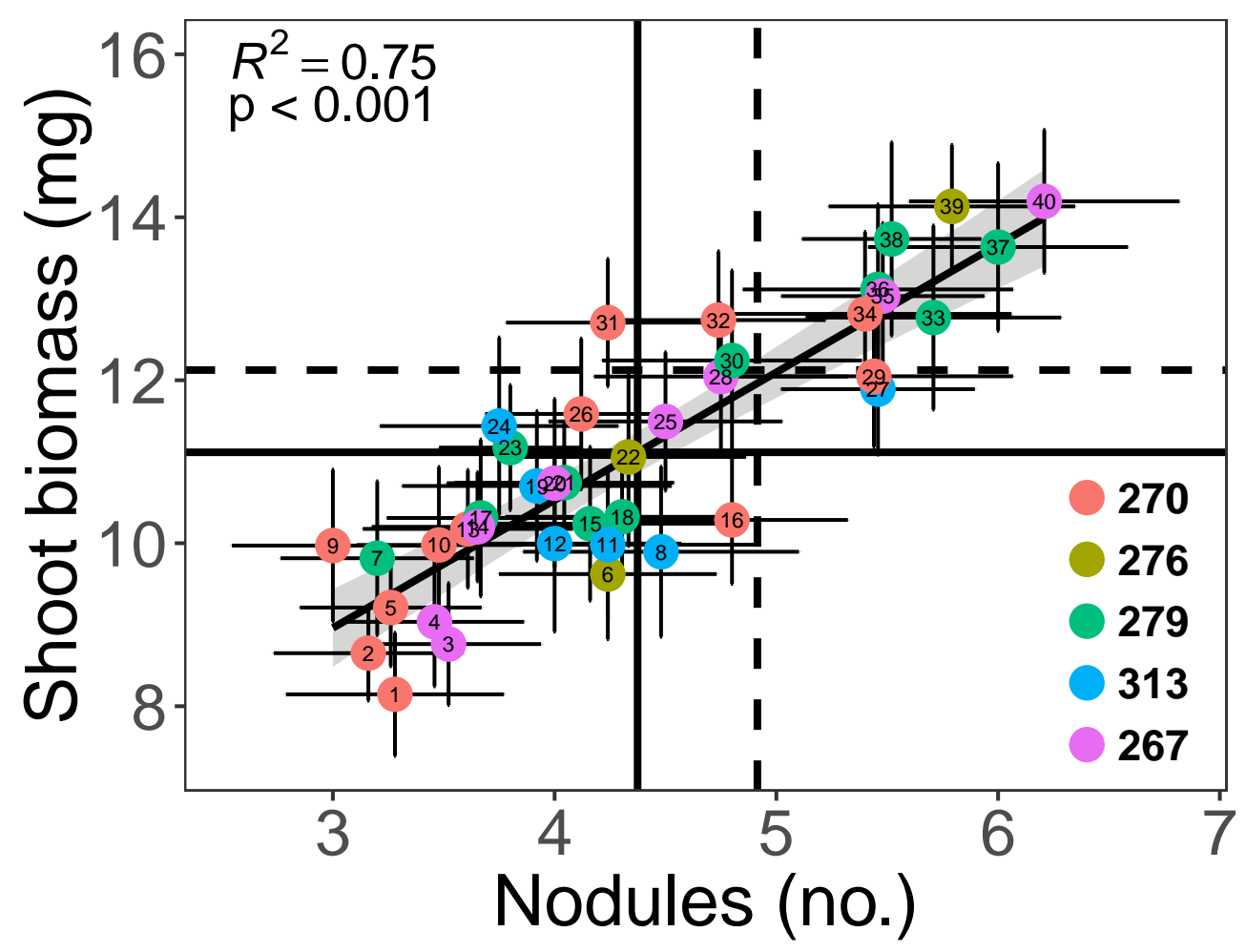

Figure S3: Fitness correlation between legumes and rhizobia, with plant fitness measured as shoot biomass and rhizobial fitness as nodule number. Each dot is the raw mean $( \pm 1 \mathrm{SE})$ for each derived Em1022 isolate averaged across all host lines. Colours show which plant line the isolate evolved on, and numbers show a unique isolate ID that is consistent across all figures. Dotted and solid lines are the raw means for quality (horizontal) or fitness (vertical) of the ancestral or derived Em1022 isolates, respectively. The $\mathrm{R}^{2}$ (adjusted) and p-values are from a linear model. 


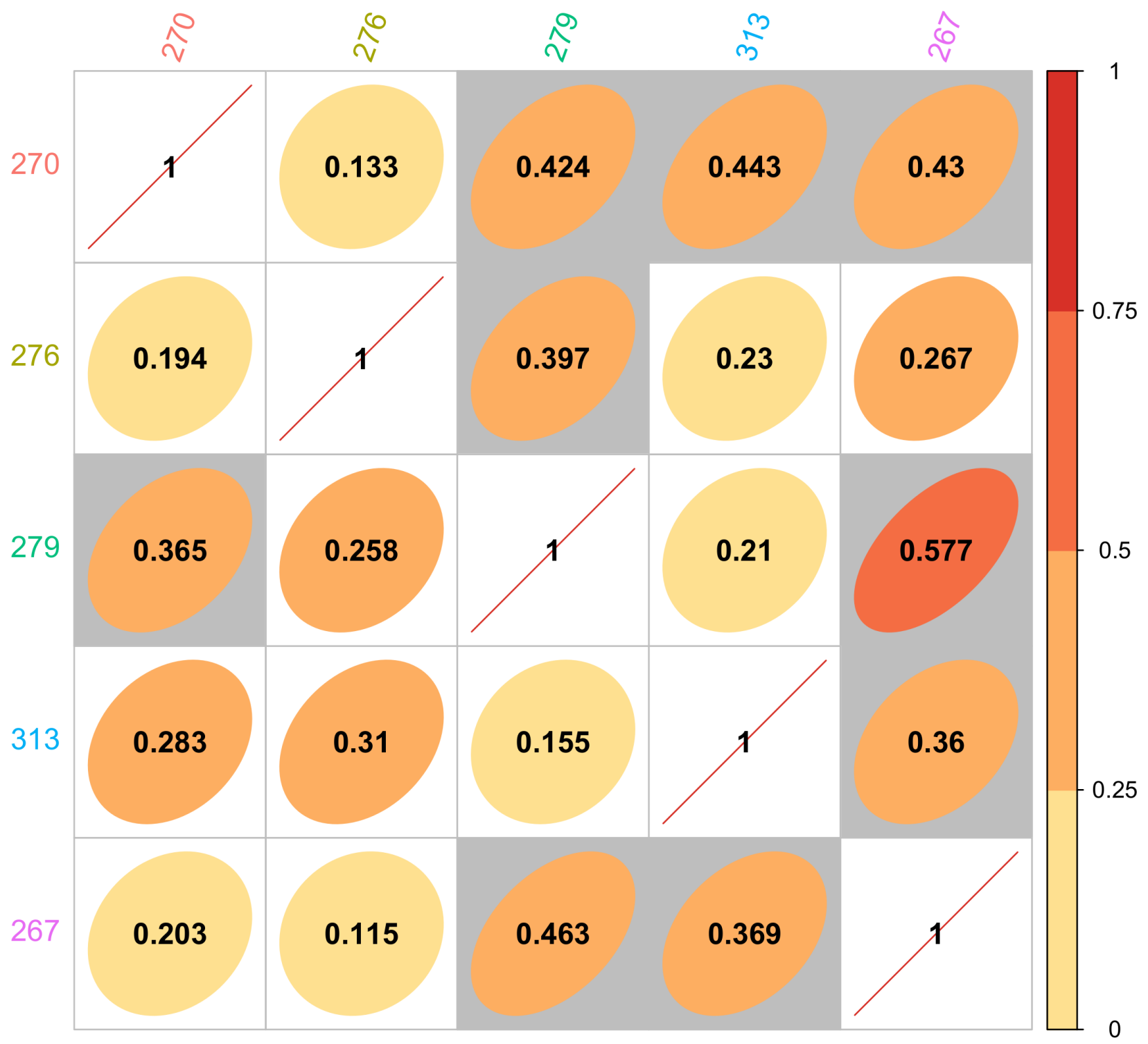

Figure S4: Correlation coefficients for isolate quality (shoot biomass, above the diagonal) and fitness (nodule number, below the diagonal) among assayed plant lines. Shaded boxes indicate significant $(\mathrm{p}<0.05)$ correlations. 

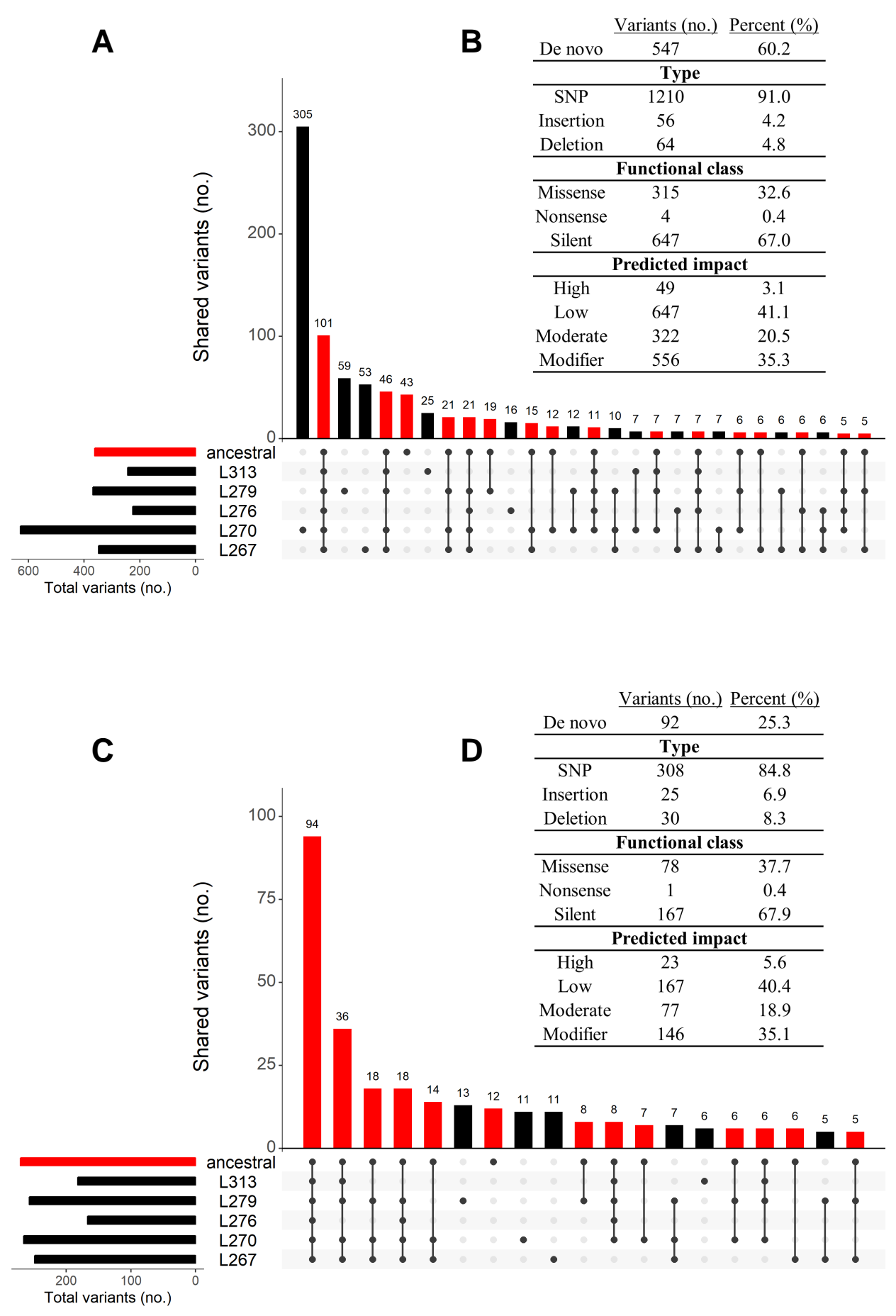

Figure S5: Number of variants unique to isolates derived on each host line, or shared among isolates derived on different host lines, or between derived rhizobia and the Em1022 ancestor. Red and black bars depict standing and de novo variants, respectively. The top panel (A \& B) shows the full variant set, after initial quality filtering, and the bottom panel (C \& D) shows the 363 variants used in the GEMMA analysis, after accounting for variants in high LD. 

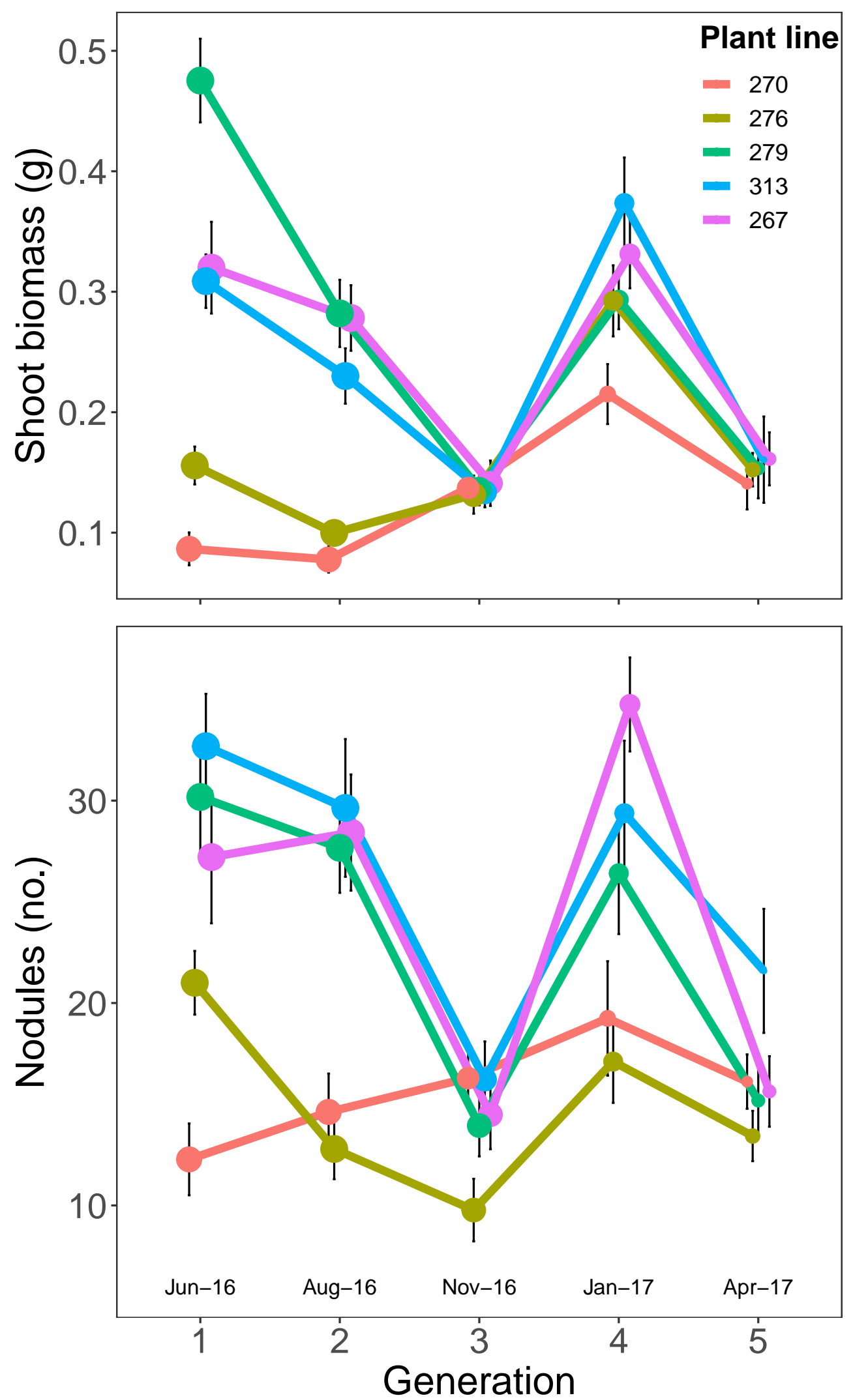

Figure S6: Mean \pm 1 SE shoot biomass (top) and nodule number (bottom) over the course of the evolution experiment. Colors are different plant lines, as in the legend. Dates at bottom are harvest dates. Size of points is proportional to sample size. 


\section{Supplementary Tables}

Table S1: Analysis of deviance (ANODEV) tables and parameter estimates for models of rhizobium quality, measured as plant aboveground biomass. In A) the model compared the quality of ancestral and derived rhizobia. In B) the model estimated the effect of shared evolutionary history on rhizobium quality; rhizobia shared evolutionary history with a plant genotype if they evolved on that plant genotype during the evolution experiment. Isolate and tray were included as random effects in all models. We square-root transformed the response variable to improve residual normality. For ANODEV tests, we determined significance using type II sums of squares, because none of the interaction terms were significant. $t$ represents the test statistic calculated for each coefficient in the linear mixed model. Bolded model terms are significant at $\mathrm{p}<0.05$.

A) Comparison of ancestral and derived rhizobia

\begin{tabular}{|c|c|c|c|c|c|c|c|}
\hline & \multicolumn{3}{|c|}{ ANODEV } & \multicolumn{4}{|c|}{ Linear mixed model summary } \\
\hline Term & $\chi^{2}$ & df & p-value & $t$ & coefficient & $\mathrm{SE}$ & p-value \\
\hline Intercept & & & & 41.299 & $\mathbf{3 . 4 3}$ & 0.083 & $<0.001$ \\
\hline Derived bacteria & 2.11 & 1 & 0.146 & -1.453 & -0.103 & 0.071 & 0.186 \\
\hline Plant genotype & 253.91 & 4 & $<0.001$ & & & & \\
\hline Line 270 & & & & -3.826 & -0.232 & 0.061 & $<\mathbf{0 . 0 0 1}$ \\
\hline Line 276 & & & & 6.829 & 0.404 & 0.059 & $<\mathbf{0 . 0 0 1}$ \\
\hline Line 279 & & & & -8.703 & -0.528 & 0.061 & $<0.001$ \\
\hline Line 313 & & & & -1.018 & -0.06 & 0.059 & 0.309 \\
\hline Isolate & 0.08 & 1 & 0.781 & & & & \\
\hline Tray & 32.42 & 1 & $<0.001$ & & & & \\
\hline
\end{tabular}

B) Effect of shared evolutionary history

ANODEV Linear mixed model summary

\begin{tabular}{l|rrr|rrrr}
\hline \multicolumn{1}{c|}{ Term } & \multicolumn{1}{c|}{$\chi^{2}$} & df & p-value & \multicolumn{1}{c}{$t$} & coefficient & \multicolumn{1}{c}{ SE } & p-value \\
\hline Intercept & & & & $\mathbf{5 8 . 3 1 7}$ & $\mathbf{3 . 3 0 3}$ & $\mathbf{0 . 0 5 7}$ & $<\mathbf{0 . 0 0 1}$ \\
Shared evolutionary history & $\mathbf{4 . 7}$ & $\mathbf{1}$ & $\mathbf{0 . 0 3}$ & $\mathbf{2 . 1 6 9}$ & $\mathbf{0 . 1 1 5}$ & $\mathbf{0 . 0 5 3}$ & $\mathbf{0 . 0 3}$ \\
Plant genotype & $\mathbf{2 2 9 . 7 4}$ & $\mathbf{4}$ & $<\mathbf{0 . 0 0 1}$ & & & & \\
$\quad$ Line 270 & & & & $\mathbf{- 3 . 5 1 4}$ & $\mathbf{- 0 . 2 2 9}$ & $\mathbf{0 . 0 6 5}$ & $<\mathbf{0 . 0 0 1}$ \\
$\quad$ Line 276 & & & & $\mathbf{6 . 6 5 6}$ & $\mathbf{0 . 4 2 5}$ & $\mathbf{0 . 0 6 4}$ & $<\mathbf{0 . 0 0 1}$ \\
$\quad$ Line 279 & & & & $\mathbf{- 8 . 3 5 2}$ & $\mathbf{- 0 . 5 4 3}$ & $\mathbf{0 . 0 6 5}$ & $<\mathbf{0 . 0 0 1}$ \\
$\quad$ Line 313 & & & & -1.01 & -0.064 & 0.063 & 0.313 \\
Isolate & 0.95 & 1 & 0.329 & & & & \\
Tray & $\mathbf{1 2 . 4 1}$ & $\mathbf{1}$ & $<\mathbf{0 . 0 0 1}$ & & & & \\
\hline
\end{tabular}


Table S2: Analysis of deviance (ANODEV) tables and parameter estimates for models of rhizobium fitness, measured by nodule number. In A) the model compared the fitness of ancestral and derived rhizobia. In B) the model estimated the effect of shared evolutionary history on rhizobium fitness; rhizobia shared evolutionary history with a plant genotype if they evolved on that plant genotype during the evolution experiment. Isolate and tray were included as random effects in all models. Both GLMMs used a Poisson error distribution to improve residual normality and homoscedasticity. For ANODEV tests, we determined significance using type II sums of squares when interaction terms were non-significant (A) or type III sums of squares when at least one interaction term was significant (B, i.e., the Line $270 \mathrm{x}$ Shared history term). $z$ represents the test statistic calculated for each coefficient in the generalized linear mixed model. Bolded model terms are significant at $\mathrm{p}<0.05$.

A) Comparison of ancestral and derived rhizobia

ANODEV

\begin{tabular}{l|rrr|rrrrr} 
& \multicolumn{3}{c}{ ANODEV } & \multicolumn{3}{c}{ Generalized linear mixed model summary } \\
\hline \multicolumn{1}{c|}{ Term } & \multicolumn{1}{c}{$\chi^{2}$} & df & p-value & \multicolumn{1}{c}{$z$} & coefficient & \multicolumn{1}{c}{ SE } & p-value \\
\hline Intercept & & & & $\mathbf{2 0 . 2 9 1}$ & $\mathbf{1 . 5 7 9}$ & $\mathbf{0 . 0 7 8}$ & $<\mathbf{0 . 0 0 1}$ \\
Derived bacteria & 1.25 & 1 & 0.264 & -1.116 & -0.082 & 0.073 & 0.264 \\
Plant genotype & $\mathbf{9 6 . 0 9}$ & $\mathbf{4}$ & $<\mathbf{0 . 0 0 1}$ & & & & \\
$\quad$ Line 270 & & & & $\mathbf{5 . 0 5 3}$ & $\mathbf{0 . 2 2 4}$ & $\mathbf{0 . 0 4 4}$ & $<\mathbf{0 . 0 0 1}$ \\
$\quad$ Line 276 & & & & $\mathbf{- 2 . 4 7 2}$ & $\mathbf{- 0 . 1 1 4}$ & $\mathbf{0 . 0 4 6}$ & $\mathbf{0 . 0 1 3}$ \\
$\quad$ Line 279 & & & & $\mathbf{- 2 . 8 4 4}$ & $\mathbf{- 0 . 1 3 8}$ & $\mathbf{0 . 0 4 9}$ & $\mathbf{0 . 0 0 4}$ \\
$\quad$ Line 313 & & & & $\mathbf{3 . 6 0 3}$ & $\mathbf{0 . 1 5 4}$ & $\mathbf{0 . 0 4 3}$ & $<\mathbf{0 . 0 0 1}$ \\
Isolate & & & & & & \\
Tray & $\mathbf{6 . 0 2}$ & $\mathbf{1}$ & $\mathbf{0 . 0 1 4}$ & & & & \\
\hline
\end{tabular}

B) Effect of shared evolutionary history

\begin{tabular}{|c|c|c|c|c|c|c|c|}
\hline & \multicolumn{3}{|c|}{ ANODEV } & \multicolumn{4}{|c|}{ Generalized linear mixed model summary } \\
\hline Term & $\chi^{2}$ & $\mathrm{df}$ & $\mathrm{p}$-value & $z$ & coefficient & SE & $\mathrm{p}$-value \\
\hline Intercept & 1003.2 & $\mathbf{1}$ & $<0.001$ & 31.673 & $\mathbf{1 . 4 6 5}$ & 0.046 & $<0.001$ \\
\hline Shared evolutionary history & 4.17 & $\mathbf{1}$ & 0.041 & 2.042 & 0.179 & 0.088 & 0.041 \\
\hline Plant genotype & 76.89 & 4 & $<\mathbf{0 . 0 0 1}$ & & & & \\
\hline Line 270 & & & & 5.426 & 0.303 & 0.056 & $<\mathbf{0 . 0 0 1}$ \\
\hline Line 276 & & & & -1.432 & -0.078 & 0.055 & 0.152 \\
\hline Line 279 & & & & -2.187 & -0.137 & 0.063 & 0.029 \\
\hline Line 313 & & & & 3.086 & 0.162 & 0.052 & 0.002 \\
\hline Plant genotype $\mathrm{x}$ Evolutionary history & 6.82 & 4 & 0.146 & & & & \\
\hline Line 270 x Shared history & & & & -2.497 & -0.306 & 0.122 & 0.013 \\
\hline Line 276 x Shared history & & & & -1.052 & -0.176 & 0.167 & 0.293 \\
\hline Line $279 \times$ Shared history & & & & -0.639 & -0.083 & 0.13 & 0.523 \\
\hline Line $313 \times$ Shared history & & & & -1.116 & -0.147 & 0.131 & 0.264 \\
\hline Isolate & 7.54 & $\mathbf{1}$ & 0.006 & & & & \\
\hline Tray & 12.45 & 1 & $<\mathbf{0 . 0 0 1}$ & & & & \\
\hline
\end{tabular}


Table S3: Genomic variants significantly associated with rhizobia quality and/or fitness. We categorised variant effects as 'cooperative' if beta scores for symbiont quality and fitness were both greater than zero (red boxes), 'defective' if beta scores were less than zero for both traits (blue boxes), 'altruistic' if beta scores were positive for symbiont quality but negative for symbiont fitness (pink boxes), or 'cheater' if beta scores were positive for symbiont fitness but negative for symbiont quality (purple boxes). Asterisks denote variants with significant effects on their 'local' hosts.

\begin{tabular}{|c|c|c|c|c|c|c|c|c|c|}
\hline \multicolumn{10}{|c|}{ Associations } \\
\hline Position & NCBI annotation & RefSeq ID & Variant type & $\begin{array}{c}\text { Host line(s) } \\
\text { of origin }\end{array}$ & $\begin{array}{l}\text { Minor allele } \\
\text { frequency }\end{array}$ & Novel? & Trait(s) & All 270276 & 279313267 \\
\hline \multicolumn{10}{|c|}{ Chromosome (N = 15) } \\
\hline 10624 & LuxR family transcriptional regulator & YP_001328801.1 & DEL & $270,279,267$ & .184 & Yes & Fitness & & \\
\hline 218304 & ATP synthase subunit beta & NP_387140.1 & SNP & 279 & .020 & Yes & Both & & \\
\hline 1377217 & chitinase & YP_001327536.1 & SNP & 279 & .020 & Yes & Fitness & & \\
\hline 1377263 & chitinase & YP_001327536.1 & SNP & anc, $270,276,279$ & .204 & No & Quality & & \\
\hline 1377284 & chitinase & YP_001327536.1 & SNP & anc, 279,313 & .082 & No & Quality & & \\
\hline 1377315 & chitinase & YP_001327536.1 & SNP & anc, $276,279,313$ & .102 & No & Fitness & & \\
\hline 1377395 & chitinase & YP_001327536.1 & SNP & 270,279 & .061 & Yes & Fitness & & \\
\hline 1377690 & chitinase & YP_001327536.1 & SNP & anc, 270 & .041 & No & Quality & & \\
\hline 1732500 & intergeneic & NA & SNP & anc, $270,276,267$ & .082 & No & Quality & & \\
\hline 1732515 & hypothetical protein & NP_385818.1 & SNP & all & .286 & No & Both & & \\
\hline 1732521 & hypothetical protein & NP_385818.1 & SNP & anc, $270,276,279,267$ & .224 & No & Both & & \\
\hline 1732545 & hypothetical protein & NP_385818.1 & SNP & anc, $270,276,279,267$ & .286 & No & Both & & \\
\hline 1732551 & hypothetical protein & NP_385818.1 & SNP & all & .306 & No & Quality & & \\
\hline 1732566 & hypothetical protein & NP_385818.1 & SNP & all & .388 & No & Quality & & \\
\hline \multicolumn{10}{|c|}{ Symbiosis plasmid A $(N=62)$} \\
\hline 12013 & hypothetical protein & WP_020479826.1 & $1 \quad$ DEL & all & .449 & No & Both & & \\
\hline 12058 & hypothetical protein & WP_020479826.1 & DEL & all & .469 & No & Fitness & & \\
\hline 352369 & potassium transporter Kup & NP_436237.1 & SNP & $270,279,267$ & .061 & Yes & Fitness & & \\
\hline 359548 & uracil-DNA glycosylase & WP_017267868.1 & SNP & anc, $270,279,267$ & .224 & No & Quality & & \\
\hline 431565 & alpha/beta hydrolase & NP_435559.2 & SNP & anc, 270 & .041 & No & Quality & & \\
\hline 431631 & alpha/beta hydrolase & NP_435559.2 & SNP & $270,276,267$ & .061 & Yes & Both & & \\
\hline 431760 & alpha/beta hydrolase & NP_435559.2 & SNP & all & .327 & No & Fitness & * & \\
\hline 432981 & adenylate cyclase & NP_435560.2 & SNP & 270 & .020 & Yes & Both & * & \\
\hline 452198 & intergeneic & NA & DEL & anc, $270,276,279,267$ & .122 & No & Quality & & \\
\hline 471937 & intergeneic & NA & INS & anc, $270,279,313,267$ & .265 & No & Both & & \\
\hline 554119 & $\mathrm{ABC}$ transporter ATP-binding protein & WP_013851031.1 & INS & all & .429 & No & Both & & \\
\hline
\end{tabular}


Table S3 continued from previous page

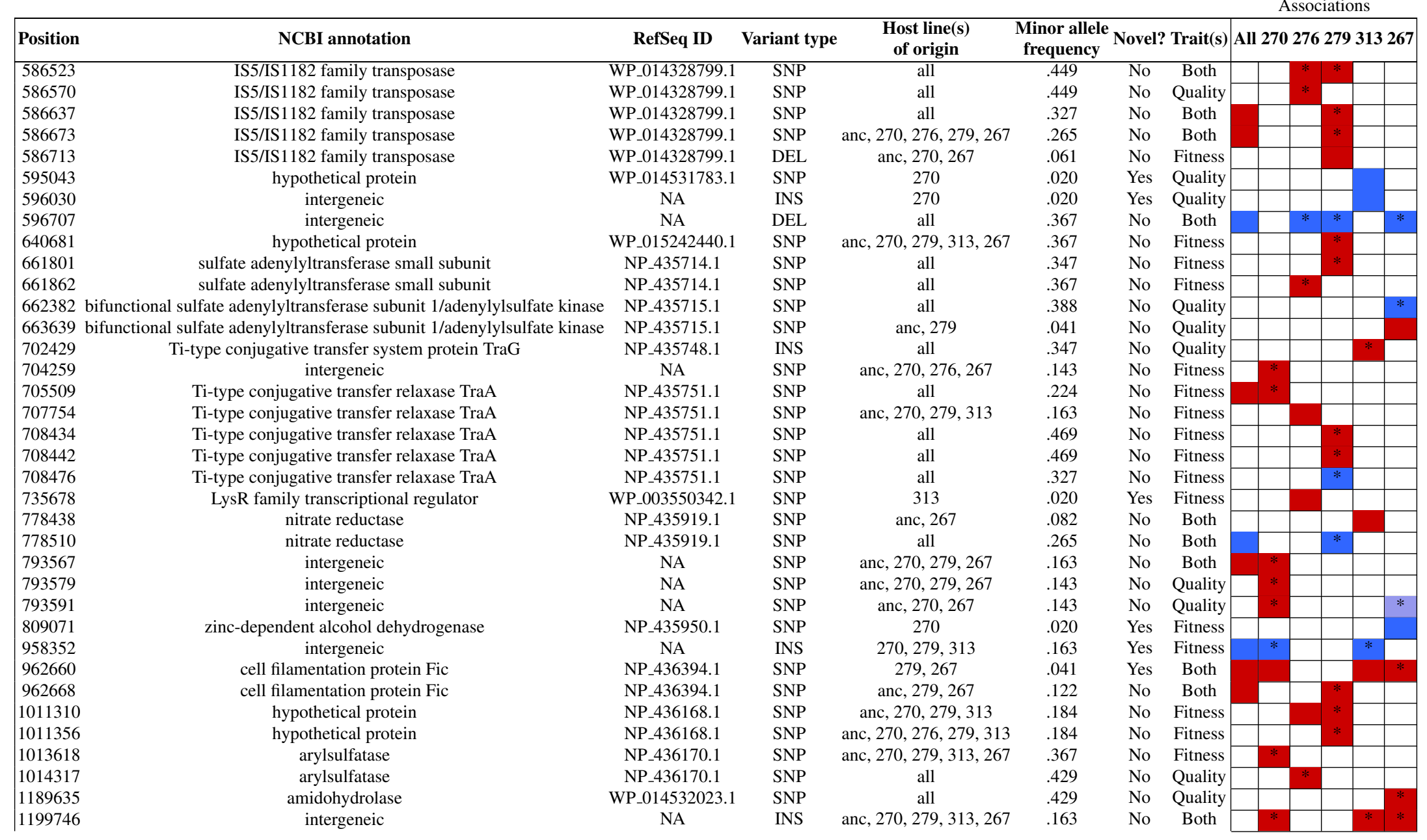


Table S3 continued from previous page

\begin{tabular}{|c|c|c|c|c|c|c|c|c|c|}
\hline \multirow{3}{*}{\begin{tabular}{|l|} 
Position \\
1200993 \\
\end{tabular}} & \multirow[b]{2}{*}{ NCBI annotation } & \multirow[b]{2}{*}{ RefSeq ID } & & \multirow[b]{2}{*}{$\begin{array}{c}\begin{array}{c}\text { Host line(s) } \\
\text { of origin }\end{array} \\
\end{array}$} & \multirow[b]{2}{*}{$\begin{array}{c}\begin{array}{c}\text { Minor allele } \\
\text { frequency }\end{array} \\
\end{array}$} & \multirow[b]{2}{*}{ Novel? } & \multirow[b]{2}{*}{ Trait(s) } & \multirow{2}{*}{\multicolumn{2}{|c|}{ All 270276279313267}} \\
\hline & & & & & & & & & \\
\hline & intergeneic & NA & SNP & anc, 270, 276, 267 & .204 & No & Fitness & & \\
\hline 1201085 & intergeneic & NA & SNP & anc, 276, 279, 267 & 102 & No & Fitness & & \\
\hline 1202352 & alpha-hydroxy-acid oxidizing protein & NP_435462.1 & SNP & all & .367 & No & Quality & & \\
\hline 1236257 & hybrid-cluster NAD(P)-dependent oxidoreductase & NP_435647.1 & SNP & anc, 270, 279, 267 & 163 & No & Quality & & \\
\hline 1236609 & hybrid-cluster NAD(P)-dependent oxidoreductase & NP_435647.1 & SNP & $270,279,267$ & .102 & Yes & Quality & & \\
\hline 1236666 & hybrid-cluster NAD(P)-dependent oxidoreductase & NP_435647.1 & SNP & anc, 279,267 & 061 & No & Fitness & & \\
\hline 1236699 & hybrid-cluster NAD(P)-dependent oxidoreductase & NP_435647.1 & SNP & anc, $270,276,313,267$ & 143 & No & Quality & & \\
\hline 1236872 & aromatic ring-hydroxylating dioxygenase subunit alpha & NP_435646.1 & SNP & all & 367 & No & Both & & \\
\hline 1238263 & LysR family transcriptional regulator & NP_435645.1 & SNP & 279,267 & .041 & Yes & Fitness & & \\
\hline 1238319 & LysR family transcriptional regulator & NP_435645.1 & SNP & anc, $270,279,313,267$ & .245 & No & Both & & \\
\hline 1238349 & LysR family transcriptional regulator & NP_435645.1 & SNP & all & 367 & No & Fitness & & \\
\hline 1238355 & LysR family transcriptional regulator & NP_435645.1 & SNP & anc, $270,279,313,267$ & .265 & No & Fitness & & \\
\hline 1238771 & LysR family transcriptional regulator & NP_435645.1 & DEL & anc, $270,279,313,267$ & .306 & No & Both & & \\
\hline \multicolumn{10}{|c|}{ Symbiosis plasmid B $(\mathbf{N}=68)$} \\
\hline 168101 & intergeneic & NA & INS & 279 & .020 & Yes & Both & & \\
\hline 189160 & intergeneic & NA & SNP & 276 & .020 & Yes & Both & & \\
\hline 195354 & hypothetical protein & WP_017267932.1 & INS & 270,267 & .041 & Yes & Both & & \\
\hline 220772 & membrane assembly protein AsmA & YP_001313723.1 & INS & anc, 279,267 & 061 & No & Fitness & & \\
\hline 227901 & intergeneic & NA & SNP & 279 & .041 & Yes & Fitness & & \\
\hline 247174 & intergeneic & NA & SNP & $270,279,267$ & 102 & Yes & Quality & & \\
\hline 247240 & intergeneic & NA & INS & all & 449 & No & Fitness & & \\
\hline 267541 & intergeneic & NA & INS & all & .408 & No & Fitness & & \\
\hline 267546 & intergeneic & NA & INS & all & .388 & No & Both & * & \\
\hline 268239 & hypothetical protein & NP_437140.1 & INS & anc, $270,276,279,313$ & .367 & No & Fitness & & \\
\hline 272788 & hypothetical protein & WP_014530542.1 & INS & 279,313 & .061 & Yes & Fitness & & \\
\hline 284773 & hypothetical protein & WP_017263565.1 & SNP & anc, $270,276,279,267$ & .347 & No & Quality & & \\
\hline 284794 & hypothetical protein & WP_017263565.1 & SNP & anc, $270,279,267$ & .347 & No & Fitness & & \\
\hline 285282 & hypothetical protein & NP_437157.1 & SNP & 276,279 & .041 & Yes & Quality & & \\
\hline 285298 & hypothetical protein & NP_437157.1 & SNP & $270,276,279$ & .061 & Yes & Both & & \\
\hline 285301 & hypothetical protein & NP_437157.1 & SNP & $270,276,279$ & .082 & Yes & Fitness & & \\
\hline 288053 & intergeneic & NA & DEL & anc, $276,279,313,267$ & .163 & No & Quality & & \\
\hline 288056 & intergeneic & NA & SNP & anc, $276,279,313,267$ & .143 & No & Quality & & \\
\hline 288081 & intergeneic & NA & DEL & 279,267 & .061 & Yes & Both & & \\
\hline
\end{tabular}


Table S3 continued from previous page

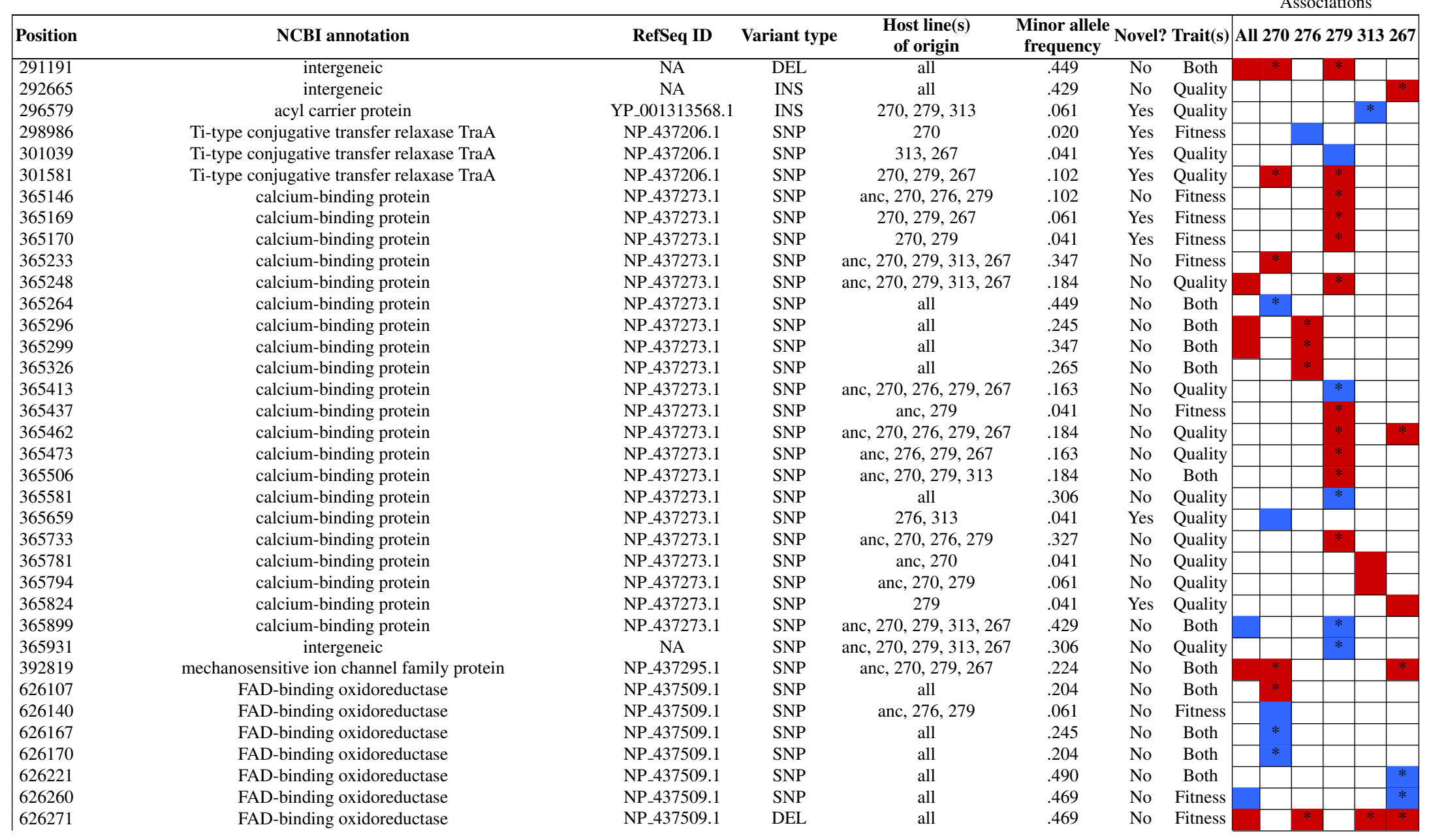


Table S3 continued from previous page

\begin{tabular}{|c|c|c|c|c|c|c|c|c|}
\hline Position & NCBI annotation & RefSeq ID & Variant type & $\begin{array}{c}\text { Host line(s) } \\
\text { of origin }\end{array}$ & $\begin{array}{l}\text { Minor allele } \\
\text { frequency }\end{array}$ & Novel? & Trait(s) & All 270276279313267 \\
\hline 626284 & FAD-binding oxidoreductase & NP_437509.1 & SNP & all & .408 & No & Both & \\
\hline 626286 & FAD-binding oxidoreductase & NP_437509.1 & SNP & all & .429 & No & Both & * \\
\hline 626293 & FAD-binding oxidoreductase & NP_437509.1 & SNP & all & .388 & No & Both & \\
\hline 626305 & FAD-binding oxidoreductase & NP_437509.1 & SNP & all & 367 & No & Both & \\
\hline 626308 & FAD-binding oxidoreductase & NP_437509.1 & SNP & all & .388 & No & Both & \\
\hline 898518 & intergeneic & NA & SNP & 270,267 & 041 & Yes & Fitness & \\
\hline 1322354 & hypothetical protein & WP_017268213.1 & SNP & 267 & .041 & Yes & Both & \\
\hline 1452970 & intergeneic & NA & SNP & 313 & .020 & Yes & Fitness & \\
\hline 1452986 & intergeneic & NA & INS & anc, 270,267 & .061 & No & Quality & \\
\hline 1471780 & cytochrome c biogenesis protein DipZ & NP_436746.1 & SNP & anc, $270,279,313,267$ & .449 & No & Fitness & \\
\hline
\end{tabular}


Table S4: Padded amplicon sequences for qPCR TaqMan Assays. Design was completed by the bioinformaticians at ThermoFisher Scientific, and was based on regions of the nodC locus that were divergent enough to distinguish between the two rhizobia strains.

\begin{tabular}{|c|c|c|c|c|c|c|}
\hline Reference sequence & Assay ID & Strain & Start pos. & End pos. ${ }^{1}$ & Padded Amplicon Sequence & bp \\
\hline \multirow[t]{5}{*}{ AE006469.1 } & AP7DPC9 & Em1021 & 479306 & 479424 & GAGATGCGCAAGACCCGTCACCACC & $1-25$ \\
\hline & & & & & GAAAGGGCGAGCAACAGTTGCCCGA & $26-50$ \\
\hline & & & & & TATTCTGTCCGACCGCGTCAAATGC & $51-75$ \\
\hline & & & & & GAGAAAAGGGCTGAGGCCGCGCAAC & $76-100$ \\
\hline & & & & & AGAGGGAGCGCTAGAAACG & $101-119$ \\
\hline \multirow{6}{*}{ AKZZ01000018.1 } & AP9HHW6 & Em1022 & 479373 & 479497 & TACATCAAATGTGAGAAAAGGGTTG & $1-25$ \\
\hline & & & & & AGGCCGCGCAACAGAGGGAGCGCTA & $26-50$ \\
\hline & & & & & GAAATGTGTCGCGGAACGTGCTGCG & $51-75$ \\
\hline & & & & & TGCCCAACGCAGTTGTTGGCGCAGA & $76-100$ \\
\hline & & & & & TACGATTTCAGCGTATCCGGGACAA & $101-125$ \\
\hline & & & & & CGGT & $126-129$ \\
\hline
\end{tabular}

${ }^{1}$ Relative to reference sequence, NC_003037.1 